\title{
A Novel and Inexpensive Approach for Force Sensing Based on FSR Piezocapacitance Aimed at Hysteresis Error Reduction
}

\author{
Arnaldo Matute $\mathbb{D}^{1},{ }^{1}$ Leonel Paredes-Madrid, ${ }^{1}$ Gelman Moreno, ${ }^{1}$ Fabián Cárdenas, ${ }^{1}$ \\ and Carlos A. Palacio ${ }_{(D)}^{2}$ \\ ${ }^{1}$ Faculty of Electronic and Biomedical Engineering, Universidad Antonio Nariño, Tunja 150001, Colombia \\ ${ }^{2}$ GIFAM Group, Faculty of Sciences, Universidad Antonio Nariño, Tunja 150001, Colombia \\ Correspondence should be addressed to Arnaldo Matute; arnaldo.matute@uan.edu.co
}

Received 11 November 2017; Revised 6 February 2018; Accepted 19 February 2018; Published 5 April 2018

Academic Editor: Dzung Dao

Copyright (C) 2018 Arnaldo Matute et al. This is an open access article distributed under the Creative Commons Attribution License, which permits unrestricted use, distribution, and reproduction in any medium, provided the original work is properly cited.

\begin{abstract}
Force-sensing resistors (FSRs) are inexpensive alternatives to load cells. They are suitable for applications where noninvasive devices are needed to measure force, stress, or pressure. However, they have been proved to be hysteresis prone and offer nonrepeatable readings due to their highly voltage-dependent electrical resistance. A piezocapacitive effect has been found as an alternative phenomenon that is able to offer force-dependent readings of capacitance with less hysteresis error. Also, this capacitance is not dependent on voltage, which also improves repeatability in force measurements. Since measuring capacitance is more expensive than resistance, the least costly conditioning circuitry is desired. An inexpensive alternative using an LM555 that oscillates depending on capacitance is here presented. Hysteresis and repeatability errors have been reduced for a widespread-used force-sensing resistor brand.
\end{abstract}

\section{Introduction}

Conductive polymer composites (CPCs), also known as quantum tunneling composites (QTCs), are manufactured by randomly dispersing conductive particles within an insulating polymer matrix. They exhibit a considerable decrement of electrical resistance when subjected to mechanical stress [1]. They are therefore of extensive use as force-sensing resistors (FSR) in robotic links, biomechanical diagnosis, touchpads, touchscreens, and industrial processes, which are demanding force-sensing accuracy with less space consumption at reduced cost [2]. Load cells have been the most linear, accurate, repeatable, and temperature-independent devices that have successfully accomplished the task of converting force to voltage $[3,4]$. Nevertheless, their cost and size have been a drawback when applications have budget and space constraints. FSRs dramatically reduce the space needs for operation as well as they are less invasive and can be easily deployed without impacting greatly the mechanical design at final application. However, they are not as linear and temperature independent as load cells, exhibit large amounts of hysteresis, when dynamic forces are exerted on them, and also drift, when the application is about static forces [5]. Moreover, its accuracy depends on the possibility of performing a correct modelling since nonlinearities are present. Thus, research endeavors are aimed at deeply understanding FSRs' behavior and also at complementing their usage with appropriate electronic conditioning circuitry and data processing techniques that could compensate errors and nonlinearities [6-9].

Hysteresis hinders the applicability of some materials in actuators [10] and also in sensors. The overall performance of FSRs is affected when dynamic forces are measured. For example, considerable linearity can be observed at FSRs by loading forces, but unloading forces are associated to a high nonlinearity and a lack of repeatability when the corresponding deformation-stress curves are studied. A complementary higher-order model for the strain-stress characteristic under unloading forces is presented in this paper in order to explain electrical hysteresis using the sensor electric model [11].

Previous works [12-16] have demonstrated that FSRs based on CPCs also embrace a capacitive effect. Their capacitance can be measured and it varies when the CPC is 
subjected to mechanical stress. Thus, measuring capacitance instead of resistance is an alternative procedure worth to assess for force sensing since the theoretical approach yields in lower hysteresis errors when capacitance readings are considered, enhancing accuracy for plenty of applications $[17,18]$. Nevertheless, measuring capacitance involves additional considerations that increase the complexity of the conditioning circuitry and, thus, the resulting costs. Thus, also a simple and cost-reduced approach for measuring capacitance is here presented.

This paper is organized as follows: Section 2 proposes an approach to mathematically explain the hysteresis phenomenon in CPCs. Section 3 combines the proposed approach of Section 2 with the electrical model of CPCs when subjected to mechanical stress. Section 4 explains and introduces the piezocapacitive effect and relates it to the previous discussion. Section 5 proposes a novel and inexpensive method to measure FSRs' capacitance. Section 6 compares the traditional with the proposed method to sense forces and exposes the experimental results. Conclusions are stated in Section 7.

\section{Deformation versus Stress Curves: $\mathbf{A}$ Proposed Complementary Approach for Unloading Stress}

Due to the capability that CPCs or QTCs have to change their electrical resistance when subjected to mechanical stress, its use as force sensors has been considered for many applications in medicine and automation. A sandwiched CPC between two electrodes, known as a traditional sandwich element (TSE) [9], is shown in Figure 1. This is the basic configuration for the Tekscan FlexiForce A201-1 according to the registered patents that explain about the electrical conduction mechanism and the way they are manufactured $[19,20]$. Rubbers, elastomers, and polydimethylsilicone (PDMS) are the preferred materials to compose the nonconductive matrix where the nanoparticles are randomly dispersed in. Nanoparticles, whose dimensions are within the range of $10^{-8}$ and $10^{-6}$ meters, are made of conductive materials such as copper or nickel. They allow electrical conduction between electrodes although thin films of the polymer matrix avoid direct physical contact among them [21-23]. Let $s$ be the average interparticle distance inside of the CPC, which changes with the applied axial stress due to polymer deformation, so it can be stated that $s=s(\sigma)$, where $\sigma$ is the mechanical stress $(\sigma)$ over the sensor-sensitive area (SSA). Let the stress $(\sigma)$ also be the ratio between the exerted force $(F)$ and the area $\left(A_{\mathrm{FSR}}\right)$ where $F$ is applied on. Loading forces are meant to reduce $s$ from an unloaded initial nondeformed state $\left(s_{0}\right)$ to a loaded lower deformed state $\left(s_{f}\right)$ due to axial stress. Unloading forces are applied when the FSR is released until $\sigma=0$. During unloading forces, the FSR recovers its nondeformed state and the average filler interparticle distance returns from $s_{f}$ to $s_{0}$, but this happens through a very different path [24].

As shown in Figure 1(a), a straight linear path is established form $s_{0}$ to $s_{f}$ as the FSR is loaded by a timeincreasing stress, but the way the interparticle separation recovers to its nonloaded state is clearly nonlinear. This hysteresis phenomenon affects also the electric behavior of the FSR since its electrical resistance depends on the instantaneous value of $s$ [11].

Let $\varepsilon$ be the strain ratio between the instantaneous interparticle separation $s$ and $s_{0}$. Equation (1) shows the linear relationship that can be established between deformation and strain in terms of $s_{0}$ for loading forces [25].

$$
s_{l}(\sigma)=s_{0}\left(1-\frac{\sigma}{M}\right) .
$$

As expected, there is a linear relation between interparticle separation and stress for loading forces which depends on the average interparticle distance at $\sigma=0, s_{0}$, and the material stiffness, $M$, both as invariant parameters. Nevertheless, for unloading forces, this linear approach is no longer valid as suggested by the aforementioned reasons. The interparticle separation recovers to its nonloaded state through a different nonlinear path. Let (2) be the relationship between $s$ and the strain during unloading forces.

$$
s_{u}\left(\varepsilon_{\mathrm{rec}}\right)=s_{f}\left(1+\varepsilon_{\mathrm{rec}}\right),
$$

where $\varepsilon_{\text {rec }}$ and $s_{u}$ are, respectively, the strain and the stress occurring when the CPC is being unloaded. It is worth to remark that $\sigma / M \neq \varepsilon_{\text {rec }}$ and $s_{l} \neq s_{u}$ due to the hysteretic phenomenon. Equation (2) describes the path that $s$ follows to recover to the nonloaded state, from $s_{f}$ to $s_{0}$. It is equivalent to the analysis needed to derive (1), but considering a positive sign since $s$ increments with the strain. When no recovery strain $\left(\varepsilon_{\text {rec }}\right)$ exists, $s=s_{f}$ and $\sigma=\sigma_{f}$. Therefore, the point in Figure 1(a) $\left(\sigma_{f}, s_{f}\right)$ is the one where loading stops and recovery path begins from. Equation (3) defines $s_{f}$ in terms of $\sigma_{f}$ which is the final and maximal stress applied when loading stops (and unloading begins).

$$
s_{f}=s_{0}\left(1-\frac{\sigma_{f}}{M}\right) \text {. }
$$

The next step to define an approach for establishing a proper relationship between interparticle separation and stress with hysteresis is about defining $\varepsilon_{\text {rec }}$. For unloading forces in the presence of hysteresis, it is straightforward to understand that (1) is not enough to represent a nonlinear relationship between strain and stress for two reasons: first, as the Young's modulus is constant for the considered material under any circumstances, a linear relation like (1) for unloading forces would yield no hysteresis at all and second, a different general linear relation considering applying a gain or an offset would be unable to describe a recovery state from $s_{f}$ to $s_{0}$ as Figure 1(a) shows. Thus, including an exponent to the expression (2) would ensure it to represent a nonlinear relation. Equation (4) combines the whole analysis discussed up to now.

$$
s_{u}(\sigma)=s_{0}\left(1-\frac{\sigma_{f}}{M}\right)\left(1+\left(\frac{a(\sigma-b)}{M}\right)^{k}\right),
$$

where $a$ and $b$ are real nonzero positive constants to be defined in a way that (1) and (4) meet in $s_{0}$ and $s_{f}$. The 


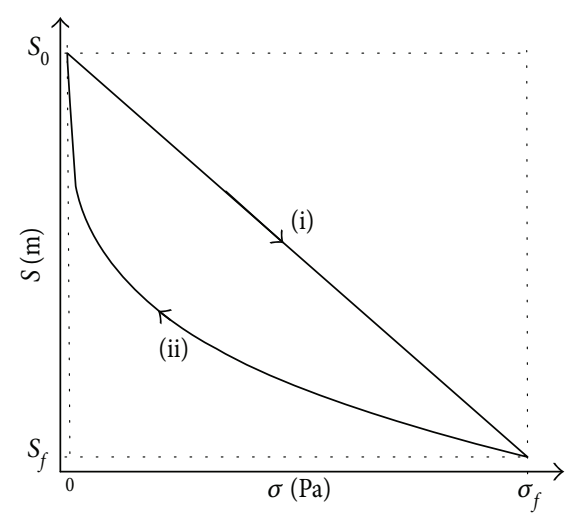

(a)

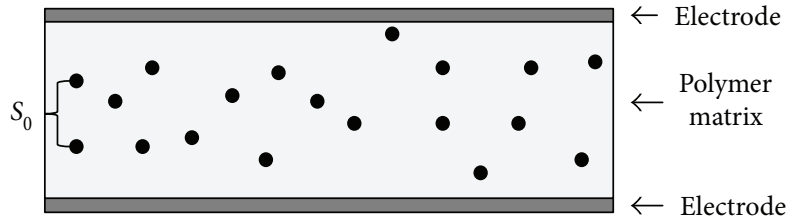

(b)

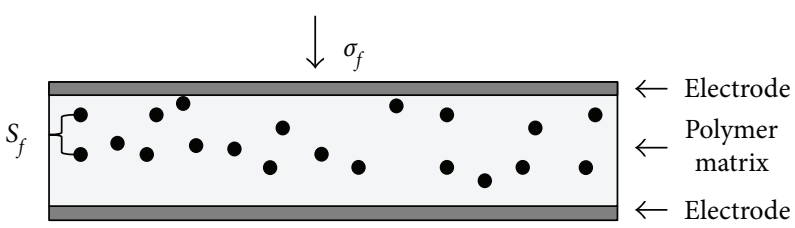

(c)

Figure 1: (a) Graph of the interparticle separation $(s)$ versus stress $(\sigma)$ for a hysteretic conductive polymer composite. Deformation (i) decreases linearly as stress increases and (ii) increases nonlinearly when the CPC is released (unloaded). (b) Scheme of polymer matrix (light gray) with conductive nanoparticles (black) sandwiched between electrodes (dark gray) not subjected to stress, where $s_{0}$ depicts the natural average interparticle separation. (c) Scheme of the same polymer matrix subjected to stress $\left(\sigma_{f}\right)$ showing that the interparticle separation has shrunk to $s_{f}$ as a consequence of stress.

constant $k$ is the exponent whose effect in this approach will be explained later in this paper. To define $a$ and $b$, it is needed to remark what (5) state for.

$$
\begin{gathered}
s_{l}(0)=s_{u}(0)=s_{0}, \\
s_{l}\left(\sigma_{f}\right)=s_{u}\left(\sigma_{f}\right)=s_{f} .
\end{gathered}
$$

After the respective algebraic operations needed from the consideration of (5), (6) is obtained. Thus, values for $a$ and $b$ can be determined:

$$
\begin{aligned}
a\left(M-\sigma_{f}\right)(-b)^{k} & =M \sigma_{f}, \\
a\left(M-\sigma_{f}\right)\left(\sigma_{f}-b\right)^{k} & =0, \\
b & =\sigma_{f}, \\
a & =\frac{M \sigma_{f}^{1-k}}{M-\sigma_{f}} .
\end{aligned}
$$

Equation (8) already considers that $k$ is an even integer in order to keep real all parameters in the equations. Combining (7) and (8) in (4), an expression for $s_{u}(\sigma)$ can be found:

$$
\begin{array}{r}
s_{u}(\sigma)=s_{0}\left(1-\frac{\sigma_{f}}{M}\right)\left(1+\left(\frac{\left(M \sigma_{f}^{1-k}\right)\left(\sigma-\sigma_{f}\right)}{M\left(M-\sigma_{f}\right)}\right)^{k}\right), \\
k=2 n, n \in \mathbb{N} .
\end{array}
$$

Equation (9) is a nonlinear approach to represent deformation at unloading forces. It accomplishes with one of the observed characteristics of hysteresis: consideration of $\sigma_{f}$, since the shape of the unloading path varies depending on the stress level reached by the exerted force. Simulations in Figure 2 show how (1) and (9) describe a hysteretic pattern when considered for loading and unloading forces, respectively. It comprises different values of $\sigma_{f}$ and $k$.

Once the proper relationships to represent deformation on stress with considerable hysteresis are established, the next step of the analysis is about its consideration in the Simmons' model [11]. It is worth to remark that the proposed approaches for $s_{l}(\sigma)$ and $s_{u}(\sigma)((1)$ and (9) are not a full model of hysteresis in CPCs. The hysteresis phenomenon is widely more complex than the stated equations, since it comprises a memory effect as already demonstrated. Moreover, it has been observed that several cycles on loading and unloading forces yield in different hysteresis paths for every cycle. That is, if (1) and (9) are considered valid and accurate enough for a first cycle, with all likelihood, they will be no longer valid for the next force cycle due to the memory effect and also due to the creep effect present in CPCs [26]. For a more accurate approach able to be considered as a model, the exponent $k$ might be considered as a time- and stress-dependent function, but this extension of the approach is beyond the scope of this paper. More thorough modeling (variational formulation, nonlinear finite elements) has been carried out for electroactive polymers (EAPs) not used for sensing though, but as actuators. However, the material EAPs are similar to 


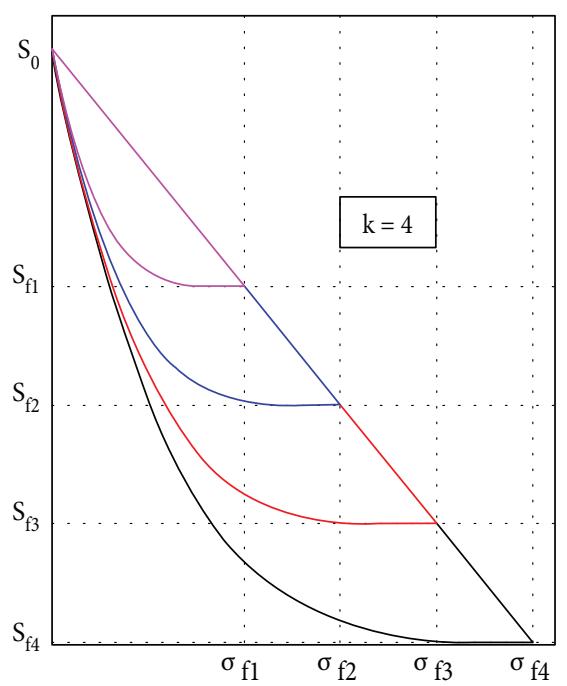

(a)

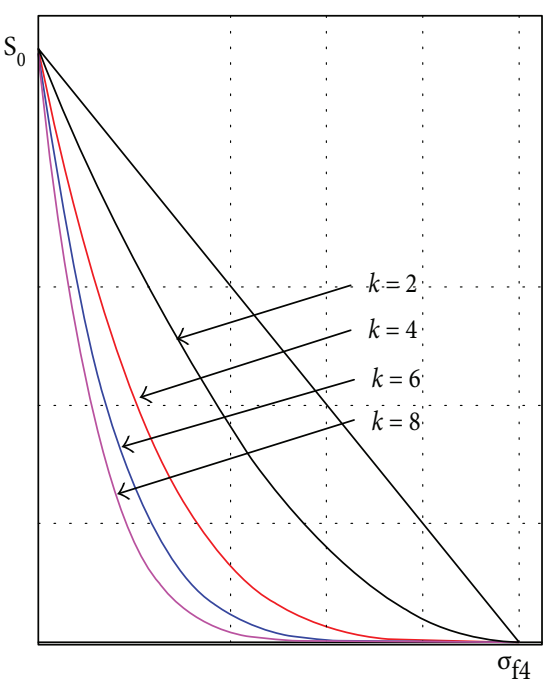

(b)

Figure 2: (a) Simulated plot for $s_{l}(\sigma)$ and $s_{u}(\sigma)$, where different values of $\sigma_{f}$ are considered. (b) Simulated plot for $s_{l}(\sigma)$ and $s_{u}(\sigma)$, where different values of $k$ are considered. MATLAB R2014 was employed to perform both simulations.

CPCs and has the same electromechanical characteristics $[27,28]$. Also, existing modelling alternatives have not been aimed at acquiring data with less hysteresis but at considering more complete models to compensate hysteresis [29]. The proposed approach will be enough to assess the electrical hysteresis as a consequence of hysteresis in the interparticle separation versus stress characteristic.

\section{Consideration of the Proposed Approach in the Simmons' Model for Force Sensing from Conductance Variation}

The Simmons' model [11] consists a set of three equations that predict the current density $(J)$ based on $s(\sigma)$ and the applied voltage $u$ in the CPC, excluding contact resistance between both electrodes and the CPC. Since CPCs are a combination of conductive particles separated by very thin layers of polymer, quantum tunneling effect happens. Electrons can flow through these thin layers which is a potential barrier even if their particle energy is not enough to classically overcome this barrier. Quantum tunneling consists in the existing probability of establishing a considerable amount that overcome a potential barrier, which is the solution of the Schrödinger equation [30]. When tunneling occurs, electrical current can be established from particle to particle. Thus, several conduction paths appear between electrodes. As stated in [30, 31], the narrower the potential barrier is, the higher the probability of the electrons to overcome the barrier through quantum tunneling is. Hence, FSR's resistance depends on this probability and this is the physical basis of the piezoresistive effect. A model of current density $J$ considering $s$ and $u$, which $E$ mainly depends on, was developed by Simmons [11] considering the multiple quantum tunneling effects occurring in a CPC. This approach was moved forward for FSRs in [31], where (11) and (12) are taken into account. This analysis complements former approaches $[6,8,9]$ where just (10) was embraced.

$J(u, s(\sigma))=\frac{3 \sqrt{2 m \varphi_{0}}}{2 s(\sigma)}\left(\frac{e}{h}\right)^{2} u \exp \left(-\frac{4 \pi s(\sigma)}{h} \sqrt{2 m \varphi_{0}}\right), \quad u \approx 0$,
$J(u, s(\sigma))=\left(\frac{e}{2 \pi h s(\sigma)^{2}}\right)\left\{\left(\varphi_{0}-\frac{e u}{2}\right) \exp \left[-\frac{4 \pi s(\sigma)}{h} \sqrt{2 m\left(\varphi_{0}-\frac{e u}{2}\right)}\right]-\left(\varphi_{0}+\frac{e u}{2}\right) \exp \left[-\frac{4 \pi s(\sigma)}{h} \sqrt{2 m\left(\varphi_{0}+\frac{e u}{2}\right)}\right]\right\}, \quad u \leq\left(\frac{\varphi_{0}}{e}\right)$,

$J(u, s(\sigma))=\frac{2.2 e^{3} u^{2}}{8 \pi h \varphi_{0} s(\sigma)^{2}}\left\{\exp \left[-\frac{8 \pi s(\sigma)}{2.96 h e u} \sqrt{2 m \varphi_{0}^{3}}\right]-\left(1+\frac{2 e u}{\varphi_{0}}\right) \exp \left[-\frac{8 \pi s}{2.96 h e u} \sqrt{2 m \varphi_{0}^{3}\left(1+\frac{2 e u}{\varphi_{0}}\right)}\right]\right\}, \quad u>\left(\frac{\varphi_{0}}{e}\right)$, 


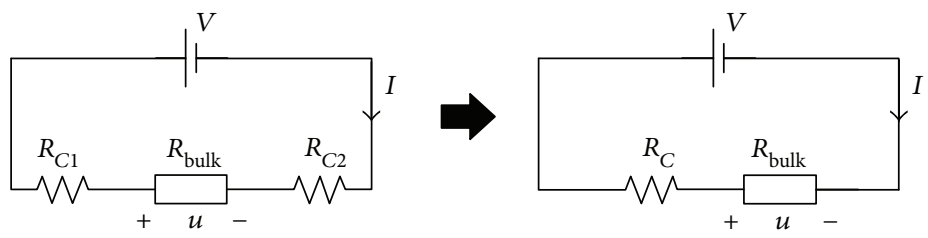

FIGURE 3: Electrical model for a FSR.

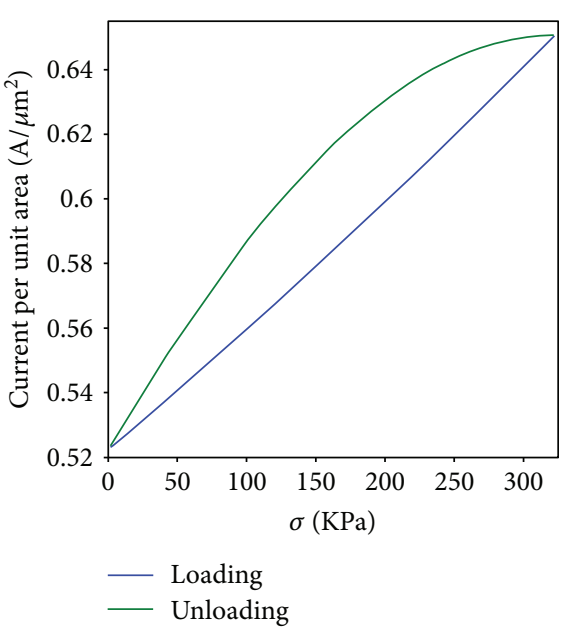

(a)

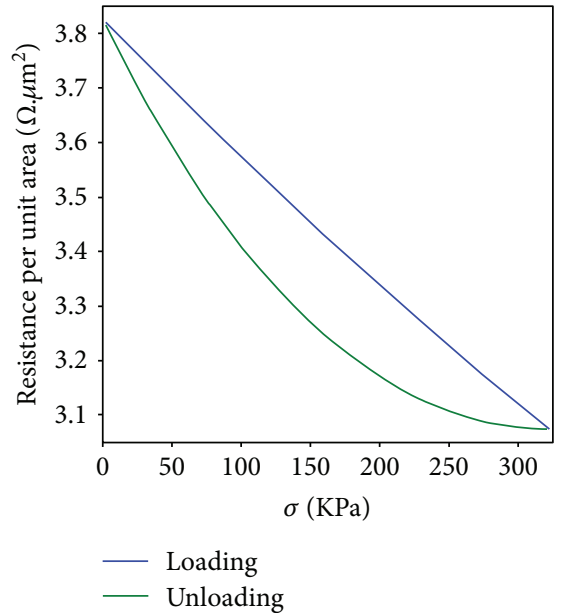

(b)

FiguRE 4: Simulation of (a) current density $(J)$ versus stress $(\sigma)$ at loading forces (blue) and at unloading forces (green). (b) Resistance per unit area $\left(R_{A}\right)$ versus stress $(\sigma)$ for the same situations. Equation (12) was considered for both simulations carried out with MATLAB R2014. Sensor invariant parameters presented in [31] were considered. They are stated in Table 1.

where $e$ is the electron's charge, $m$ its mass, $\varphi_{0}$ is the height of the potential barrier, and the constant $\hbar=h / 2 \pi$, being $h$ the Planck's constant. Equations (10) through (12) show two aspects to be assessed. The dependence on $s(\sigma)$ and on $u$ are as follows:

(i) Dependence on $s(\sigma)$ : the shape of $J$ when the FSR is subjected to increasing stress (loading) will be different to the shape when unloading since $J\left(u, s_{l}(\sigma)\right) \neq$ $J\left(u, s_{u}(\sigma)\right)$. This leads to understand that mechanical hysteresis provokes electrical hysteresis.

(ii) Dependence on $u$ : let $V$ be the constant voltage applied by the conditioning circuitry on the FSR. Let $I$ be the current that flows through the FSR and $R_{C}$ the sum of the contact resistance that the interface of the CPC and electrodes provides to the whole resistance of the FSR (see Figure 3). It is straightforward to understand that $u=V-R_{C} I$, but it has been already stated that $R_{C}$ is a function of stress. This is $R_{C}=R_{C}$ $(\sigma)[8,32,33]$. Therefore, $u$ is also a function of stress $u=u(\sigma)$ and this makes the current density $J$ highly dependent on stress. Hence, the characteristic $J$ versus $\sigma$ is also significantly affected by hysteresis as Figure 4(a) shows. This has been simulated considering the parameters shown in Table 1.

As well as the $J$ versus $\sigma$ one, the characteristic resistance per unit area, $R_{A}$ versus $\sigma$, where $R_{A}=u / J$, also presents
TABLE 1: Parameters for simulation in Figure 4.

\begin{tabular}{lc}
\hline Parameter & Value \\
\hline$M$ & $4.73 \mathrm{MPa}$ \\
$\sigma_{f}$ & $322 \mathrm{kPa}$ \\
$U$ & $2 \mathrm{~V}$ \\
$s_{0}$ & $4.418 \mathrm{~nm}$ \\
$\varphi_{0}$ & $0.223 e$ \\
$\varepsilon_{r}$ & 7 \\
$\varepsilon_{0}$ & $8.85 \cdot 10^{-12} \mathrm{C}^{2} / \mathrm{Nm}^{2}$ \\
\hline
\end{tabular}

considerable hysteresis due to the already-studied hysteresis observed in the $s$ versus $\sigma$ characteristic. Figure 4(b) shows that the behavior of resistance per unit area is as linear as expected. This is desirable to measure pressure or force based on resistance. Moreover, Figure 4(a) also presents the desired linear increment of density current with stress. Current through the whole CPC in axial direction is the product of the effective conductive area $A$ and density current, which also depend on stress. Thus, $A=A(\sigma)$. For comparison purposes, the hysteresis error will be defined in a manner that $A(\sigma)$ will not affect calculations. Hence, hysteresis error can be defined in terms either of $J$ or $I$, which is $I=I(u, s(\sigma))=A(\sigma) J(u, s(\sigma))$. The formulation for the hysteresis error for any physical quantity is defined by 


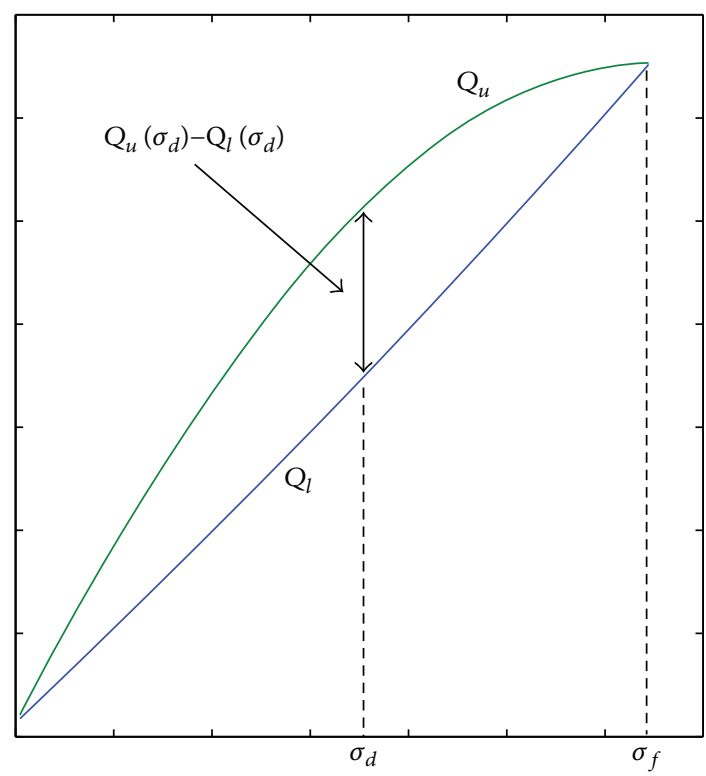

Figure 5: Terms to calculate HE regardless of the involved physical quantity.

$$
\mathrm{HE}=100 \% \frac{Q_{u}\left(\sigma_{d}\right)-Q_{l}\left(\sigma_{d}\right)}{Q\left(\sigma_{f}\right)}
$$

where $Q$ is the physical quantity to calculate its hysteresis error (HE), $Q_{u}$ is $Q$ at unloading forces, $Q_{l}$ is $Q$ at loading forces, $\sigma_{d}$ is the stress where the maximal difference between $Q_{u}$ and $Q_{l}$ occurs, and $\sigma_{f}$ is the already-defined final stress. Figure 5 depicts terms in (13). HE will be considered hereafter in terms of percentage.

\section{Consideration of the Proposed Approach in the Capacitive Model}

CPCs present the already described piezoresistive effect due to the quantum tunneling conduction that occurs when conductive particles are separated by thin insulating films. However, this is not the only electrical phenomenon that can be observed in CPCs and specifically in FSRs. Between two conductive particles, which are separated by an insulating material, also, a capacitive effect takes place. Confronted particles are able to accumulate electrical load and to establish an electrical field as shown in Figure 6. The usage of FSRs' based on piezocapacitive effect for several applications has been reported by [13-15], and strong evidence of capacitance existence in FSRs has been published in [12] where phase shifting between voltage and current was observed when the FSR was fed with AC power.

The combined piezocapacitive effect with quantum tunneling conduction result in the $\mathrm{R}-\mathrm{C}$ circuit is depicted in Figure 7. Resistances, originated from quantum tunneling conduction, can be combined in parallel if the tunneling conduction occurs between particles that are at the same potential. Then, the resulting resistances happen to be connected in series: vertically placed particles where quantum tunneling occurs successively as a conduction path that exists from electrode to electrode through the whole polymer matrix. These quantum tunneling resistances are connected in series. Thus, a total resistance of the FSR can be obtained but its calculation as a circuit is not straightforward. Resistance per unit area is the result of dividing the voltage in the CPC, $u$, by the current density $J$ from (10) through (12). This yields an expression that depends on $s(\sigma)$ and as a consequence of quantum tunneling, on $u$. Similarly, capacitances in the CPC can also be combined in series and in parallel in order to obtain an equivalent capacitance for the FSR. Equation (14) is a valid manner of calculating capacitance in piezoresistive sensors [34]. For the sake of fair comparison and regarding to the fact that the behavior of the effective conductive area is beyond the scope of this paper, let $C_{A}$ be the capacitance per unit area of the CPC, defined in (15).

$$
\begin{aligned}
C & =\frac{\varepsilon_{r} \varepsilon_{0} A(\sigma)}{s(\sigma)}, \\
C_{A} & =\frac{\varepsilon_{r} \varepsilon_{0}}{s(\sigma)},
\end{aligned}
$$

where $\varepsilon_{0}$ is the dielectric permittivity of vacuum and $\varepsilon_{r}$ is the relative dielectric permittivity of rubber, that is, the material polymer matrix is composed of. Thus, expressions for the resistance per unit area as well as for the capacitance per unit area are finally obtained in the set from (16) through (18) and in (15), respectively.

$$
\begin{aligned}
R_{A}(s(\sigma)) & =\frac{2 s(\sigma)}{3 \sqrt{2 m \varphi_{0}}}\left(\frac{h}{e}\right)^{2} \exp \left(\frac{4 \pi s(\sigma)}{h} \sqrt{2 m \varphi_{0}}\right), \quad u \approx 0, \\
R_{A}(u, s(\sigma)) & =\frac{2 \pi h s(\sigma)^{2} u}{\left\{\left(\varphi_{0} e-\left(e^{2} u / 2\right)\right) \exp \left[-((4 \pi s(\sigma)) / h) \sqrt{2 m\left(\varphi_{0}-(e u / 2)\right)}\right]-\left(\varphi_{0} e+\left(e^{2} u / 2\right)\right) \exp \left[-(4 \pi s(\sigma) / h) \sqrt{2 m\left(\varphi_{0}+(e u / 2)\right)}\right]\right\}}, \quad u \leq\left(\frac{\varphi_{0}}{e}\right), \\
R_{A}(u, s(\sigma)) & =\frac{(16)}{2.2 e^{3} u\left\{\exp \left[-((8 \pi s(\sigma)) / 2.96 h e u) \sqrt{2 m \varphi_{0}^{3}}\right]-\left(1+\left(2 e u / \varphi_{0}\right)\right) \exp \left[-(8 \pi s / 2.96 h e u) \sqrt{2 m \varphi_{0}^{3}\left(1+\left(2 e u / \varphi_{0}\right)\right)}\right]\right\}}, \quad u>\left(\frac{\varphi_{0}}{e}\right)
\end{aligned}
$$



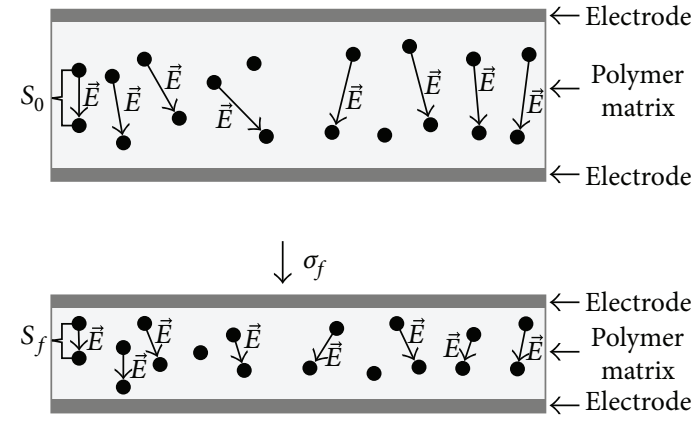

FIGURE 6: Diagram of FSR where possible electrical fields between particles are depicted. A couple of confronted conductive particles can behave as capacitor. Although not depicted, electrical field between electrodes and neighboring particles also exists.

It is worth to recall that (16) through (18) are the result of dividing $u$ by (10) through (12). Hence, (16) yields in a voltage-independent expression, only valid when $u$ is small. As long as $u$ increases, its effect on the resistance is noticeable and (17) and (18) are voltage and stress dependent. Figure 8 shows how $R_{A}$ and $C_{A}$ behave when a loading-unloading pattern of forces is exerted on a FSR. For the simulation shown, the applied voltage $V$ is larger than $\varphi_{0} / e$, so (18) is valid to calculate $R_{A}$.

Numerical values of hysteresis errors of (13) can be calculated based on simulations of Figure 8. Table 2 presents the values of $\mathrm{HE}$ for the simulations performed in Figures 9(a) and 9 (b). As already mentioned, $R_{A}$ is highly dependent on $u$ whereas $C_{A}$ does not depend on $u$ as shown in (14) and (15). Therefore, Table 2 shows different HE for $R_{A}$ whereas the $\mathrm{HE}$ for $C_{A}$ remains unchanged regardless of the voltage level, $u$.

In all cases, the HE is lower for capacitance than the corresponding HE for the resistance in the simulation. The reason for this reduction of hysteresis error in capacitance readings relies on the fact that $s(\sigma)$ is not in the argument of an exponential function neither in (14) nor in (15) since piezocapacitance is a nonquantum phenomenon, but a classic consequence of confronting two conductive plates with a dielectric in the middle. Conversely, $s(\sigma)$ exists in the argument of exponential functions in (16) through (18) that describe $R_{A}$, which exacerbates rheological hysteresis.

Capacitance's expressions are also voltage independent, which could improve repeatability towards changes in voltage (either in $V$ or in $u$ ), which are common in FSR, as $u=u(\sigma)$ due to the fact that $u=V-R_{C} I$ and $R_{C}=R_{C}$ $(\sigma)$. Therefore, the proposal of measuring capacitance instead of resistance for force sensing seems attractive due to its likely hysteresis error reduction and also its lower repeatability error due to the simulations' results shown in Figure 8 and Table 2. Nevertheless, measuring capacitance is more complicated and expensive than measuring resistance, but the likely error reduction could pay off the cost increment in circuitry. The next section explains how a low-cost circuit used to measure capacitance was designed.

\section{Capacitance Measurement with a LM555 in Modified Astable Operation}

Measuring capacitance is not as straightforward as measuring resistance, which can be done with inexpensive and simple circuitry [35, 36]. Nevertheless, several strategies to measure capacitance have been successfully implemented with the purpose of force sensing as well as what ParedesMadrid et al. and Damilano et al. have done [12, 14]. However, the most concerning drawback of those approaches was costs. Although it can be understood that more accurate force reading based on capacitance can payoff the boost in costs, it is also interesting to lower them as much as possible. A LM555 single timer in astable operation was considered to be an alternative strategy to measure capacitance due to its low cost. Figure 9(a) shows the traditional astable configuration for the LM555.

In astable operation, the output of the depicted circuit in Figure 9(a) is a switched response whose frequency and duty cycle depends on the value of resistances $R_{A}$ and $R_{B}$ and on capacitance $C 1$. Thus, the output frequency $f$ can be related to force as the applied stress modifies sensor's capacitance. Equation (19) shows the relationship between frequency and capacitance in LM555 astable operation whose derivation can be consulted in [37]. This analysis is performed considering the periods of charge and discharge of $\mathrm{Cl}$.

$$
f=\frac{1.44}{2 R C 1} \text {. }
$$

If the value of $R$ is constant, frequency and capacitance are in an inverse-proportional relationship. Hence, measuring FSR piezocapacitance can be achieved by considering the circuit in Figure 9(a), where $C 1$ is replaced by the FSR itself (capacitance, CFSR, and resistance, RFSR), as depicted in Figure 9(b). However, it must be recalled that the circuital model for the FSR is a resistance (RFSR) and a capacitance (CFSR) in parallel, which cannot be avoided. The method to propose is about measuring piezocapacitance aiming to reduce hysteresis error due its nonquantum electrical behavior. Neither piezoresistance nor piezoimpedance would lead to a less hysteretic response due its quantum resistive component that makes exponential relations appear. The new condition modifies the typical analysis done in [37] and, therefore, (19). Hence, the needed approach to derive the equivalent expression to (19) considering the modification shown in Figure 9(b) is presented next.

5.1. When Capacitor CFSR Charges. According to the analysis performed in [37] but considering that the capacitance $C 1$ is replaced by an FSR, the resulting circuit in the charge period is shown in Figure 10. Thus, the voltage in the capacitor CFSR $\left(V_{\text {CFSR }}(t)\right)$ is derived using the general solution for the obtained RC circuit:

$$
V_{\mathrm{CFSR}}(t)=V_{\mathrm{CFSR}}(\infty)+\left(V_{\mathrm{CFSR}}(0)-V_{\mathrm{CFSR}}(\infty)\right) e^{\left(-t / R_{\mathrm{th}} \mathrm{CFSR}\right)},
$$




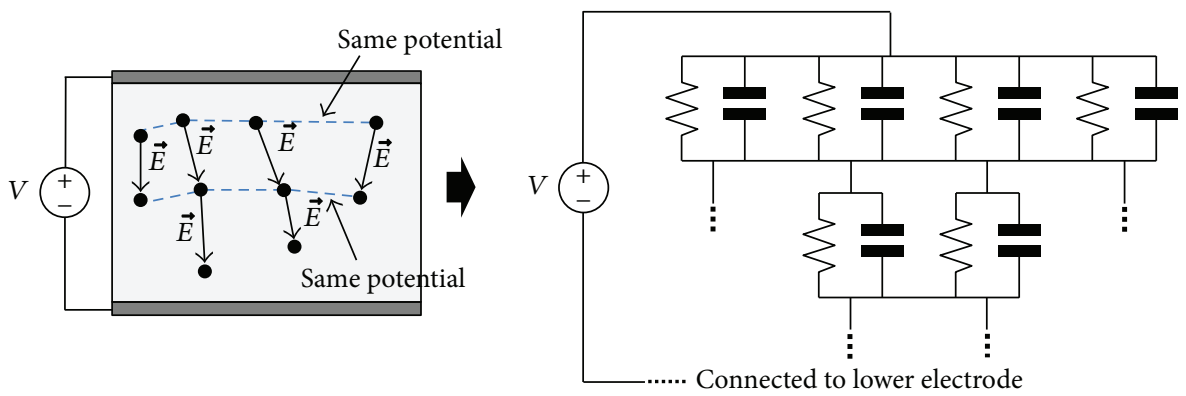

Figure 7: Resulting RC circuit in a FSR subjected to a potential $V$.

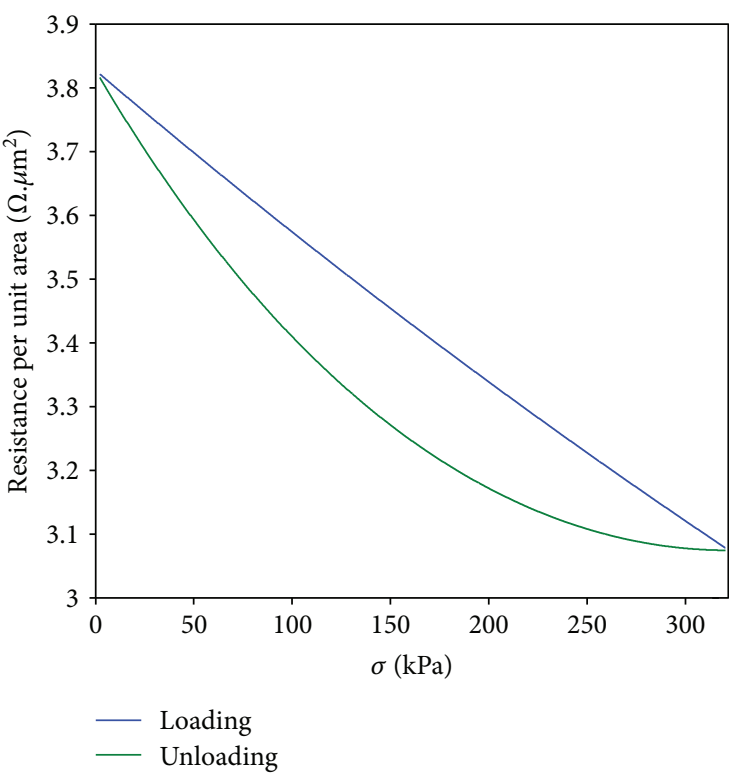

(a)

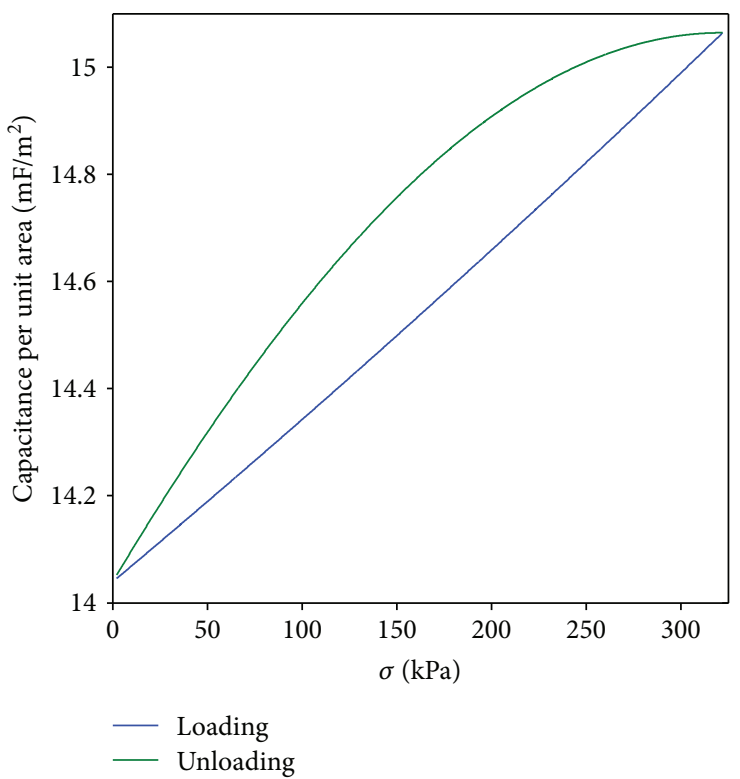

(b)

FIGURE 8: (a) Resistance per unit area versus stress and (b) capacitance per unit area versus stress. Both graphs present considerable hysteresis due to the already-known hysteresis provided by $s(\sigma)$. For $(a)$, parameter values shown in Table 1 were considered.

where $V_{\text {CFSR }}(\infty)$ is $V_{\text {CFSR }}(t \rightarrow \infty)$, that is, the voltage that CFSR reaches when it is fully charged; $V_{\text {CFSR }}(0)$ is $V_{\text {CFSR }}$ $(t=0)$, that is, the voltage across CFSR when the charging period has just begun; and $R_{\mathrm{th}}$ is the Thèvenin equivalent resistance seen from CFSR.

As also done in the derivation of expression found in [37], $V_{\text {CFSR }}(\infty)$ is the voltage divider seen on CFSR and $V_{\text {CFSR }}(0)=\mathrm{Vcc} / 3$. It can be observed that $R_{\mathrm{th}}$ is the parallel of $2 R$ and RFSR. So, (20) can be rewritten as

$$
\begin{aligned}
V_{\mathrm{CFSR}}(t)= & \frac{\mathrm{VccRFSR}}{2 R+\mathrm{RFSR}} \\
& +\left(\frac{\mathrm{Vcc}}{3}-\frac{\mathrm{VccRFSR}}{2 R+\mathrm{RFSR}}\right) e^{\left(-t /\left(\left(\left(2 R^{*} \mathrm{RFSR}\right) /(2 R+\mathrm{RFSR})\right) \mathrm{CFSR}\right)\right)} .
\end{aligned}
$$

TABLE 2: Hysteresis error for resistance and capacitance at several levels of $u$ calculated from the simulations of Figure 8 .

\begin{tabular}{lcc}
\hline$u(V)$ & $\begin{array}{c}\text { HE for resistance } \\
\text { per unit area }\end{array}$ & $\begin{array}{c}\text { HE for capacitance } \\
\text { per unit area }\end{array}$ \\
\hline 3 & $2.21 \%$ & $1.73 \%$ \\
2 & $5.95 \%$ & $1.73 \%$ \\
1 & $10.58 \%$ & $1.73 \%$ \\
\hline
\end{tabular}

Let $\tau_{H}$ be the time period that CFSR takes to reach $(2 / 3) \mathrm{Vcc}$ :

$$
\begin{aligned}
V_{\mathrm{CFSR}}(t)= & \frac{2}{3} \mathrm{Vcc}=\frac{\mathrm{VccRFSR}}{2 R+\mathrm{RFSR}} \\
& +\left(\frac{\mathrm{Vcc}}{3}-\frac{\mathrm{VccRFSR}}{2 R+\mathrm{RFSR}}\right) e^{\left(-\tau_{H} /\left(\left(\left(2 R^{*} \mathrm{RFSR}\right) /(2 R+\mathrm{RFSR})\right) \mathrm{CFSR}\right)\right)}
\end{aligned}
$$


After working on (22), the value found for $\tau_{H}$ corresponds to the expression in

$$
\tau_{H}=\ln \left(\frac{2 R-2 \mathrm{RFSR}}{4 R-\mathrm{RFSR}}\right) \frac{2 R^{*} \mathrm{RFSR}}{2 R+\mathrm{RFSR}} \text { CFSR. }
$$

5.2. When Capacitor CFSR Discharges. The equivalent analysis is followed with the resulting circuit shown in Figure 11.

Considering $V_{\mathrm{CFSR}}(0)=(2 / 3) \mathrm{Vcc}, V_{\mathrm{CFSR}}(\infty)=0$, and $R_{\mathrm{th}}$ the parallel of $R$ and RFSR, (20) can be rewritten as

$$
V_{\mathrm{CFSR}}(t)=\left(\frac{2}{3} \mathrm{VCC}\right) e^{\left.\left(-t /\left(\left(R^{*} \mathrm{RFSR}\right) /(R+\mathrm{RFSR})\right) \mathrm{CFSR}\right)\right)} .
$$

Let $\tau_{L}$ be the time period that CFSR takes to discharge up to $\mathrm{Vcc} / 3$ again:

$$
V_{\mathrm{CFSR}}(t)=\frac{\mathrm{Vcc}}{3}=\left(\frac{2}{3} \mathrm{Vcc}\right) e^{\left(-\tau_{L} /\left(\left(\left(R^{*} \mathrm{RFSR}\right) /(R+\mathrm{RFSR})\right) \mathrm{CFSR}\right)\right)}
$$

$$
\tau_{L}=\ln (2) \frac{R^{*} \text { RFSR }}{R+\text { RFSR }} \text { CFSR. }
$$

The output frequency of the circuit is

$$
f=\frac{1}{\tau_{H}+\tau_{L}}=\frac{1}{\left((\ln ((2 R-2 \mathrm{RFSR}) /(4 R-\mathrm{RFSR})))\left(\left(2 R^{*} \mathrm{RFSR}\right) /(2 R+\mathrm{RFSR})\right)+\ln (2)\left(\left(R^{*} \mathrm{RFSR}\right) /(R+\mathrm{RFSR})\right)\right) \mathrm{CFSR}} .
$$

The duty cycle (DC) of the output signal is

$$
\mathrm{DC}=\frac{\tau_{H}}{\tau_{H}+\tau_{L}}=\frac{(\ln ((2 R-2 \mathrm{RFSR}) /(4 R-\mathrm{RFSR})))\left(\left(2 R^{*} \mathrm{RFSR}\right) /(2 R+\mathrm{RFSR})\right)}{(\ln ((2 R-2 \mathrm{RFSR}) /(4 R-\mathrm{RFSR})))\left(\left(2 R^{*} \mathrm{RFSR}\right) /(2 R+\mathrm{RFSR})\right)+\ln (2)\left(\left(R^{*} \mathrm{RFSR}\right) /(R+\mathrm{RFSR})\right)} .
$$

Analyzing (23), restrictions for the values of $R$ have to be set in order to ensure $\tau_{H}$ to be nonnegative. Natural logarithm's argument has to be

$$
\begin{gathered}
\frac{2 R-2 \text { RFSR }}{4 R-\text { RFSR }}>1, \\
R<\frac{\text { RFSR }}{4} .
\end{gathered}
$$

Equation (30) is the conclusion for the aforesaid restriction about the natural algorithm argument. This means that if (30) is not satisfied, the output of the circuit in Figure 9(b) will not oscillate. Let (31) be the relation between $R$ and RFSR, where $N \in(4, \infty)$, as (30) demands.

$$
R=\frac{\mathrm{RFSR}}{N}
$$

Substituting (31) in (23) and (26), the defining ratio between $\tau_{H}$ and $\tau_{L}$ (32) is obtained. Figure 12 shows its graph.

$$
\frac{\tau_{H}}{\tau_{L}}=\frac{\ln ((2-2 N) /(4-N))(2 N)(N+1)}{\ln (2)(N+2)} .
$$

The minimal value of the ratio $\tau_{H} / \tau_{L}$ for $N>4$ is 24.37 . This means that the duty cycle of the output signal cannot be smaller than $24.37 / 25.37=0.96$. An output signal with a duty cycle of at least $96 \%$ is unpractical for sensing. This is quite close to the point of no oscillation at all. Physical explanation behind this mathematical approach consists in the fact that $\tau_{L}$ is too short due to the highly fast discharge of CFSR. In Figure 11, it can be seen that during the discharge period, CFSR discharges through the paralleled resistances $R$ and RFSR, which is very low and demands considerable amount of current. This is a concerning pitfall for performing force sensing with this method that could be solved by modifying the circuit in Figure 9(b) including a series diode (D1) with $R_{B}$ as Figure 13 shows.

The existence of $D 1$, considered as an ideal diode with no voltage drop, does not modify the analysis done in the charging period and it remains unchanged. Equation (21) though is still valid for the circuit in Figure 13. However, the analysis for the discharging period changes considerably because $D 1$ disconnects $R$ from the equivalent circuit. The existence of $D 1$ modifies the circuit in Figure 11 in a manner that CFSR is discharged only through RFSR and no longer the parallel between $R$ and RFSR, as Figure 14 shows.

Just with the exception of a new time constant, the analysis that led to (24) remains unchanged. Considering the new situation, (20) is rewritten as follows: 


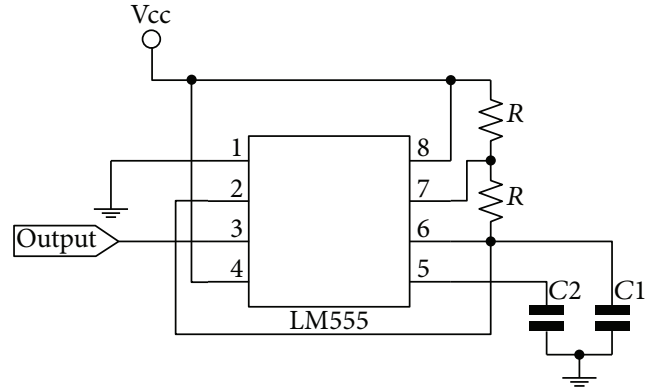

(a)

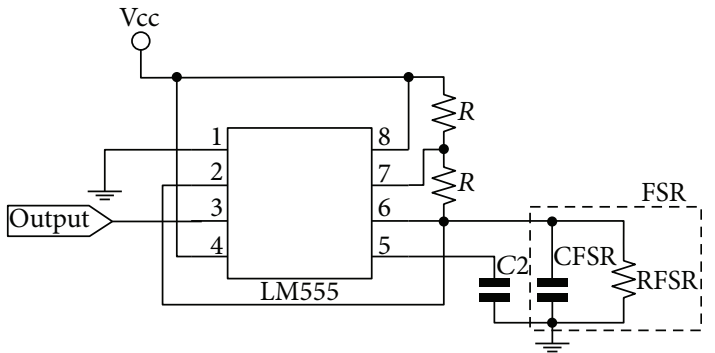

(b)

Figure 9: (a) Typical circuit of LM555 in astable operation. (b) LM555 in astable operation with FSR replacing capacitance C1.

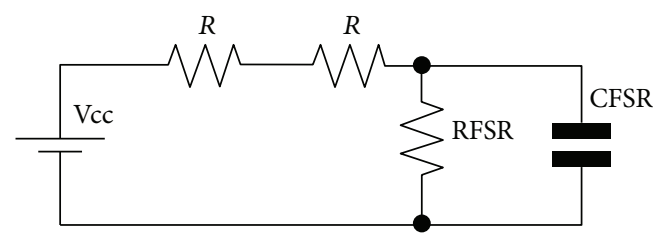

FIGURE 10: Equivalent RC circuit obtained considering the LM555 in astable configuration modified according to Figure 8(b), when CFSR is being charged.

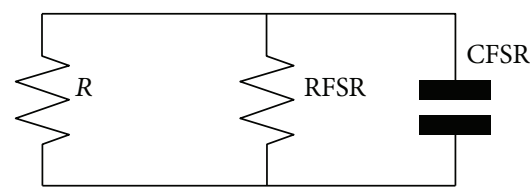

FIGURE 11: Equivalent RC circuit obtained considering the LM555 in astable configuration modified according to Figure 9(b), when CFSR is being discharged.

$$
V_{\mathrm{CFSR}}(t)=\left(\frac{2}{3} \mathrm{VCC}\right) e^{\left(-t /\left(\operatorname{RFSR}{ }^{*} \mathrm{CFSR}\right)\right)}
$$

The new value of $\tau_{L}$ is

$$
\tau_{L}=\ln (2) \mathrm{RFSR}^{*} \mathrm{CFSR} .
$$

Equations (27), (28), and (32) change as follows with the inclusion of $D 1$. As analyzed with (32), Figure 15 is the graph

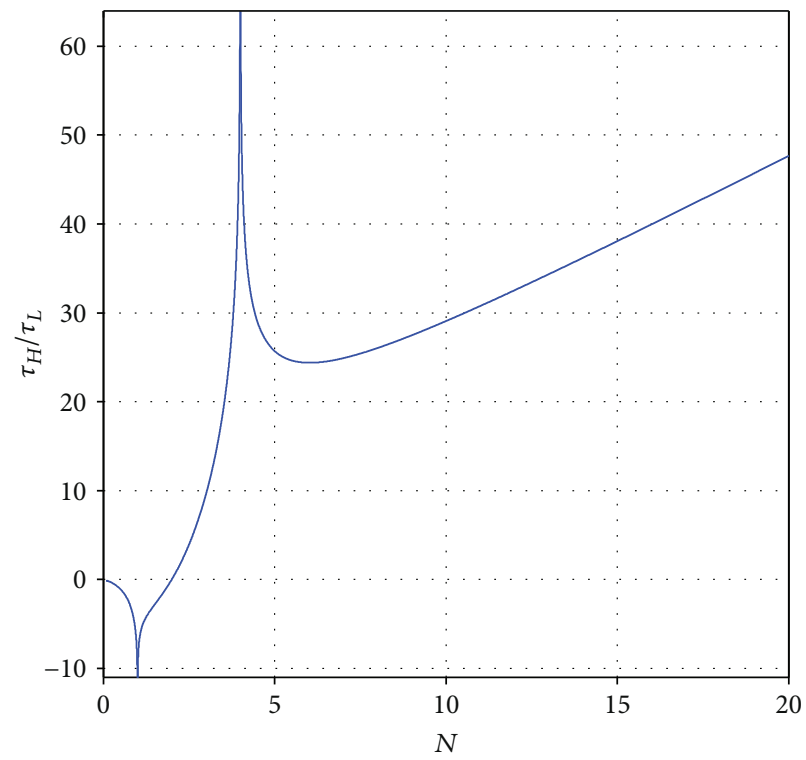

Figure 12: $\tau_{H} / \tau_{L}$ ratio versus $N$.

of (37), where there is no local minimum beyond $N=4$, so almost any value of $\tau_{H} / \tau_{L}$ can be found. Due to the fact that $\lim _{N \rightarrow \infty} \tau_{H} / \tau_{L}=2$, considering the definition of (37), the smallest duty cycle possible is $66.6 \%$ and any value above this can be achieved by selecting the proper value of $R$. This configuration offers a wider range for the duty cycle and allows to work away from the point where the output of the circuit stops oscillating.

$$
\begin{aligned}
f & =\frac{1}{\left((\ln ((2 R-2 \mathrm{RFSR}) /(4 R-\mathrm{RFSR})))\left(\left(2 R^{*} \mathrm{RFSR}\right) /(2 R+\mathrm{RFSR})\right)+\ln (2) \mathrm{RFSR}\right) \mathrm{CFSR}}, \\
\mathrm{DC} & =\frac{(\ln ((2 R-2 \mathrm{RFSR}) /(4 R-\mathrm{RFSR})))\left(\left(2 R^{*} \mathrm{RFSR}\right) /(2 R+\mathrm{RFSR})\right)}{(\ln ((2 R-2 \mathrm{RFSR}) /(4 R-\mathrm{RFSR})))\left(\left(2 R^{*} \mathrm{RFSR}\right) /(2 R+\mathrm{RFSR})\right)+\ln (2) \mathrm{RFSR}}, \\
\frac{\tau_{H}}{\tau_{L}} & =\frac{(\ln ((2-2 N) /(4-N)))(2 N)}{\ln (2)(N+2)} .
\end{aligned}
$$




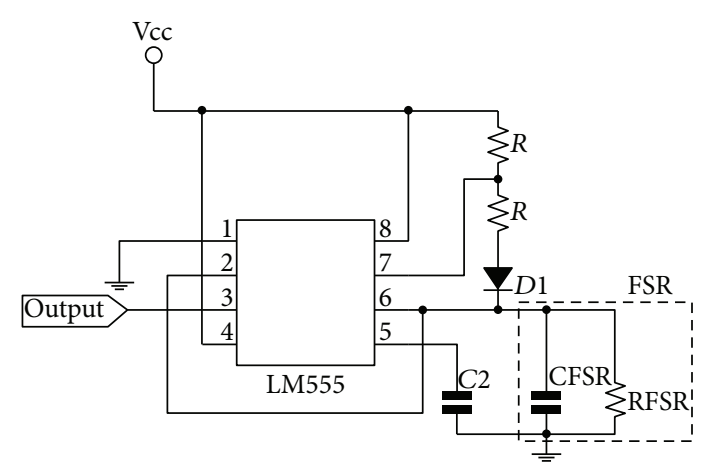

FIGURE 13: LM555 in astable operation with FSR replacing capacitance $C 1$ and diode in series with $R_{B}$.

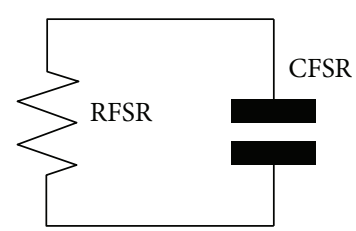

FIGURE 14: Modified circuit in Figure 11 due to the existence of $D 1$.

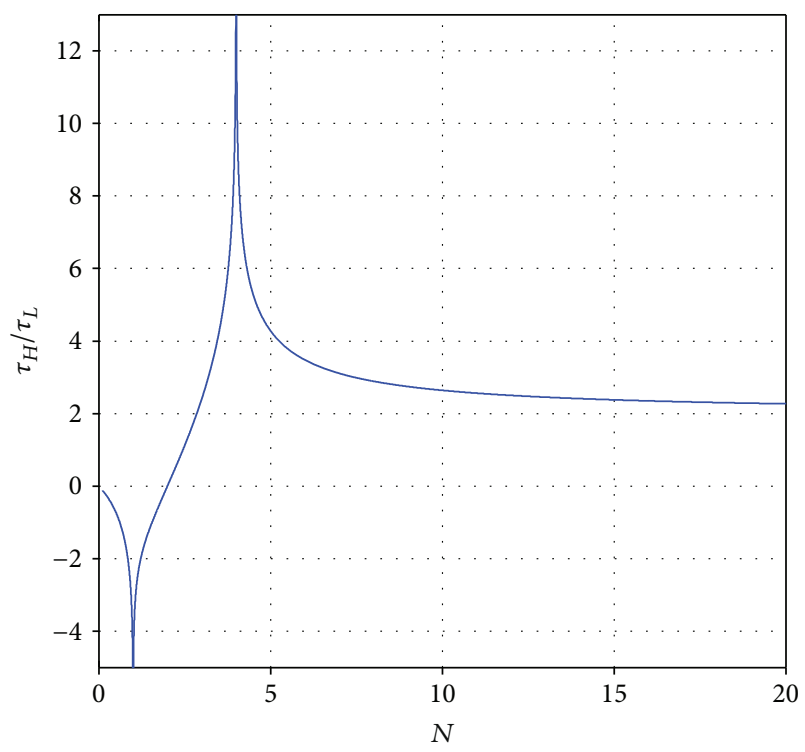

FIgURE 15: $\tau_{H} / \tau_{L}$ ratio versus $N$ considering the existence of $D 1$.

Finally, an offset capacitance (C_off) has been added in parallel to the FSR. Since the relationship between frequency and capacitance is inverse, if CFSR happens to be too low, the resulting frequency would be too high. With a minimal capacitance, a maximal frequency could be calculated and thus, a specific range of frequencies can be established. This is important for the logic digital circuit that will measure the output frequency. The resulting circuit is shown in Figure 16. The output is connected to a logic circuit based on Arduino, whose programmed algorithm decodes

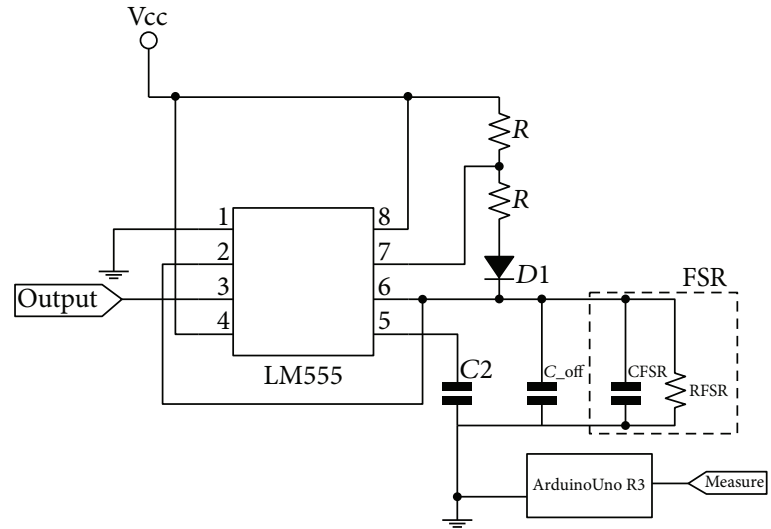

FIGURE 16: Final conditioning circuit for force sensing based on capacitance and frequency.

frequency and duty cycle and also multiplexes either C_off and the FSR itself, regarding the force range that it is intended to measure.

It is worth to remark that the presence of RFSR in the circuit cannot be neither eliminated nor diminished since this is a fundamental characteristic of FSRs. A possible concern that the parallel RFSR may cause would be the possibility to bypass CFSR when the applied stress is high, which makes RFSR to be considerably reduced. However, it must be remarked that RFSR is both stress and voltage dependent, and therefore, in order to avoid bypassing CFSR, the sourcing voltage Vcc has to be set to a reduced value so that amid the large applied stress, RFSR remains large enough. Equations (16) through (18) show that the lower the applied voltage is, the higher the resistance becomes [38]. From Figure 16, the largest voltage connected to the FSR is two-thirds of the sourcing voltage, and thus, RFSR is consequently held within acceptable limits. Given that $\mathrm{Vcc}=5 \mathrm{~V}$ and considering the highest voltage across the FSR, $2 / 3 \mathrm{Vcc}=3.33 \mathrm{~V}$, there are no concerns about bypassing CFSR.

Once the conditioning circuit has been fully defined, the next testing process made was about applying different forces and registering the output frequencies for each applied force. Thus, a force versus frequency characteristic can be graphed and analyzed. A versatile workbench has been considered in order to gather the data for the forthcoming experiments. A stepper motor was accommodated to exert controlled forces on FSR specimens though a spring. Loading and unloading forces could have been applied in order to acquire the frequency data for several force levels. Detailed information about the workbench can be found in $[31,36]$.

Figure 17(a) shows that a linear fitting for the graphed characteristic is not appropriate, which suggests that this approach is not to be the most adequate for the obtained data. Although data are approximately well distributed on both sides of the line graph, the fitting process yielded a poor goodness of fit $\left(R^{2}\right)$ of 0.9432 , what seems not attractive since this proposal is about improving error percentages which are below 11\% (see Table 2). The reasons that make the linear 


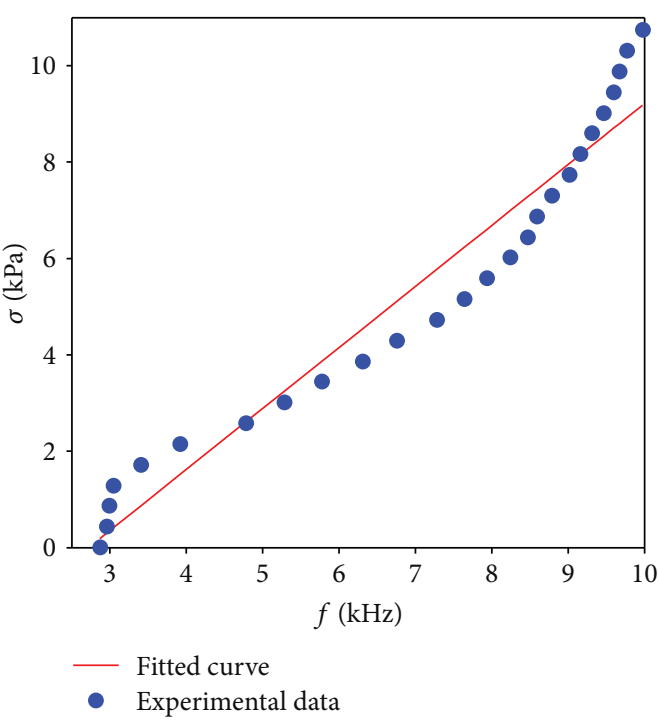

(a)

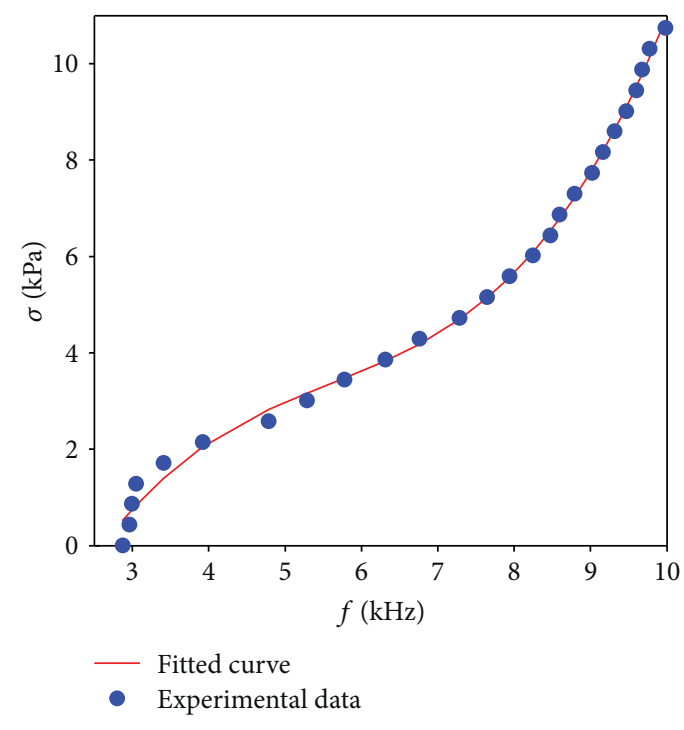

(b)

FIGURE 17: (a) Linear fitting for frequency versus force characteristic with $R^{2}=0.9432$. (b) Third-order polynomial fitting for frequency versus force characteristic with $R^{2}=0.9974$.

approach not suitable although it was theoretically expected are listed next:

(i) Conductive area influence: it is worth to recall that either the FSR total resistance or its total capacitance is the product of $R_{A}$ or $C_{A}$, respectively, with the effective conductive area, which is the gathering of all the formed conductive paths and depends highly on stress, but is worth to state that its influence reduces linearity to all relationships between physical quantities it multiplies [31].

(ii) RFSR influence: as (35) shows, even if $R \ll \mathrm{RFSR}$, the dependence on RFSR still exists. Equations (16) through (18) are worth to be evaluated in order to recall how nonlinear the relation between resistance and stress is. This remaining dependence on RFSR affects linearity considerably.

However, Figure 17(b) is a third-order polynomial approach that fits better the data.

It is worth to recall that not only that this endeavor of measuring capacitance with the proposed method is mainly for the sake of cost but also that it provides considerable flexibility: It allows determining the frequency range to consider with the proper selection of $C$ _off and high-speed multiplexing. More accurate solutions, such as the ones presented here, offer a more linear characteristic to measure capacitance, but their main drawbacks are cost [12] and flexibility [14]. Hence, a cost of inaccuracy is paid if this inexpensive conditioning circuit is considered, but the cost seem not to be too high when the aim of considering capacitance is to lower an error from theoretically less than $11 \%$ to an error below $2 \%$. The third-order polynomial approach proposes to pay a cost of computational processing instead of the cost of inaccuracy, which seems to be more attractive considering that

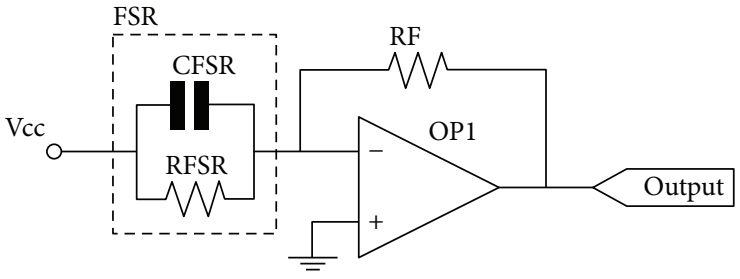

FIGURE 18: Conditioning circuitry for force measuring based on FSR through the traditional method. The contribution of CFSR is not considered. RF is the feedback resistance, which is constant.

linearity error introduced may be greater than the reduction of error to expect with this proposed method.

\section{Experimental Error Comparison}

6.1. Hysteresis Error. Four (04) Tekscan FlexiForce A201-1 sensors were considered to be the sample for the experimental procedure. Hysteresis errors were measured using the traditional method and also the proposed capacitive technique. Figure 18 shows the simple conditioning circuit to measure a physical quantity related to the FSR's resistance. In order to avoid working with negative voltage signals, $\mathrm{Vcc}$ is set to a negative value. Capacitance CFSR does not play any role since Vcc is direct current. The proposed method considers the circuit already studied and shown in Figure 16. It is worth to recall that the output measurable physical quantity related to force for the proposed method is frequency. Conversely, for the traditional method, the output physical quantity related to force that has to be considered is the output voltage of the amplifier. The relation between RFSR and the output voltage is given as follows:

$$
V_{\text {output }}=-\frac{\mathrm{RF}}{\mathrm{RFSR}} \mathrm{Vcc} \text {. }
$$


TABle 3: Hysteresis error for both the traditional and proposed methods.

\begin{tabular}{lcc}
\hline Sensor & $\mathrm{HE}_{\mathrm{TM}}$ & $\mathrm{HE}_{\mathrm{PM}}$ \\
\hline 1 & $20.06 \%$ & $16.58 \%$ \\
2 & $23.36 \%$ & $14.23 \%$ \\
3 & $21.42 \%$ & $15.67 \%$ \\
4 & $28.54 \%$ & $18.77 \%$ \\
Average error & $23.35 \%$ & $16.31 \%$ \\
\hline
\end{tabular}

The circuit in Figure 16 was used to acquire force measurements based on capacitance. Loading and unloading forces were applied to four (04) sensors in order to calculate hysteresis error according to (13). The test bench described in [31] was employed again. It is worth to remark that these measurements were done to the same sensors with both methods. The obtained errors are presented in Table 3. The conditioning circuit in Figure 18 was powered up with $\mathrm{Vcc}=-5 \mathrm{~V}$ and its $\mathrm{RF}=20 \mathrm{k} \Omega$. Figure 19 shows the readings of frequency and voltage that were acquired with each method.

As expected, in Table 3, it is clear that considering the theoretical approach presented in Sections 2 and 3, the errors for the proposed method are considerably lower than the errors that the traditional method presents. Hysteresis error of the traditional method $\left(\mathrm{HE}_{\mathrm{TM}}\right)$ is similar to the reported errors calculated in [28]. However, according to simulated results in Table 2, two-digit errors were not expected. The following aspects have to be taken in account to explain hysteresis errors above the simulated results:

(i) Effective conductive area: simulated results just embrace resistance per unit area as well as capacitance per unit area, not considering the effect of conductive areas. Experimental measurements rely on resistance and capacitance already affected by conductive areas. Thus, a comparison between data in Tables 2 and 3 has to consider that the effect of conductive areas is not taken into account in both analyses. As stated in [31], effective conductive area depends on the applied stress and it has been studied for loading forces. A model of the effective conductive area at unloading forces has not been proposed, but the data presented in this paper suggests that the effective conductive area presents also additional hysteresis, which boosts the total HE.

(ii) Test bench hysteresis: it is also true that the test bench (springs and stepper motor) provides hysteresis beyond the amount predicted at simulations, which may exaggerate the $\mathrm{HE}$ as Table 3 shows. However, since both $\mathrm{HE}_{\mathrm{TM}}$ and $\mathrm{HE}_{\mathrm{PM}}$ were calculated based on data acquired with the same test bench at the same circumstances, comparison may be carried out.

6.2. Repeatability Error. Repeatability error was also evaluated in this comparison through (39). Two manners of calculating errors were employed. Since repeatability error is about static loading, dead weights were considered instead of the stepper motor, which was appropriate for dynamic loading. The data to calculate errors in both ways is gathered according to the following process:

(i) Loading the sensor with a $200 \mathrm{~g}$ dead weight to acquire the measurements of voltage or frequency, depending on the method to assess. Then, retire the mass pattern.

(ii) Repeat the previous step five times. A set of five values of voltage or frequency has been collected for the same load applied.

(iii) Repeat the two previous steps with another dead weight to acquire another data set in a different loading condition.

With the acquired data, $\mathrm{RE}_{1}$ and $\mathrm{RE}_{2}$ can be calculated:

$$
\begin{aligned}
& \mathrm{RE}_{1}=100 \% \frac{\left|X_{\mathrm{Max}}-X_{\mathrm{Min}}\right|}{\bar{X}}, \\
& \mathrm{RE}_{2}=100 \% \frac{\sqrt{\left(\left((X-\bar{X})^{2}\right) / n\right)}}{\bar{X}},
\end{aligned}
$$

where $X_{\mathrm{Max}}$ is the maximal value in the acquired set, $X_{\mathrm{Min}}$ is the minimal one, $\bar{X}$ is the mean value for the same set, $X$ is any value that belongs to the set, and $n$ is the quantity of values that the set has. It is worth to remark that $R_{2}$ is the ratio between the standard deviation of the set and its mean, in percentage. Both errors have been calculated for the same four sensors that the hysteresis error was calculated on. Also, two loading conditions were considered: $200 \mathrm{~g}$ and $400 \mathrm{~g}$. In all cases and with all analyses done, repeatability error dramatically decreases with the proposed method in comparison with the traditional one. This was observed regardless of the loading condition ( $200 \mathrm{~g}$ or $400 \mathrm{~g}$ ) or the error formula indicator ((39)) to consider for comparison. The errors in Table 4 outperform in all circumstances to those on Table 5. This was expectable due to the capacitance's nondependence on voltage. Since piezoresistance in CPCs is a quantum tunneling phenomenon, it is evidently dependent on voltage as theoretically predicted in [11] and demonstrated in [30]. As seen in Table 2, different errors are exhibited, for readings based on conductance, depending on the considered voltage to apply. If voltage is not constant, resistance neither is. Therefore, repeatability is affected. This is worth to remark due to the widespread use of voltage dividers as conditioning circuit $[8,29,39]$ that do not guarantee constant voltage on the FSR. Even when the considered circuit for this work (Figure 18) is not a voltage divider but a single OpAmp inverter circuit, the lack of repeatability still appears due to other causes. However, the circuit in Figure 18 is a better solution in comparison with voltage dividers, which is discouraged due to the aforementioned reasons. The proposed method that comprises the circuit in Figure 16 has outperformed even the OpAmp inverter regarding to repeatability error due to the independence of piezocapacitance on voltage. 


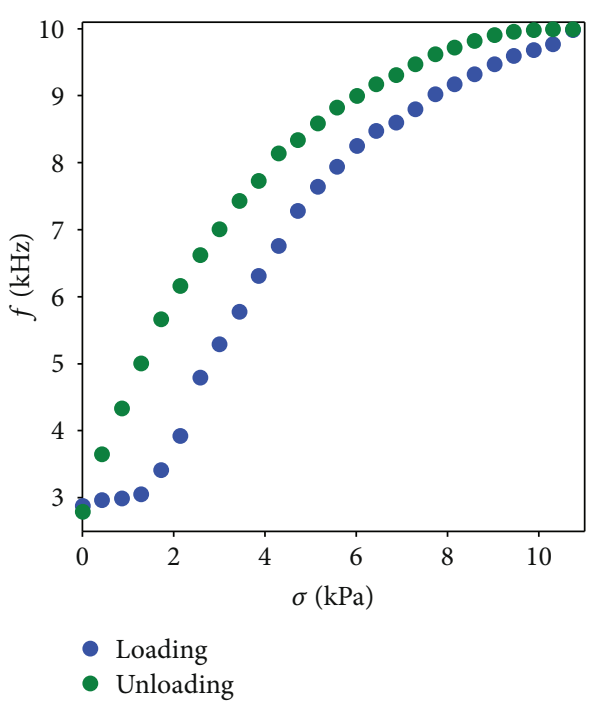

(a)

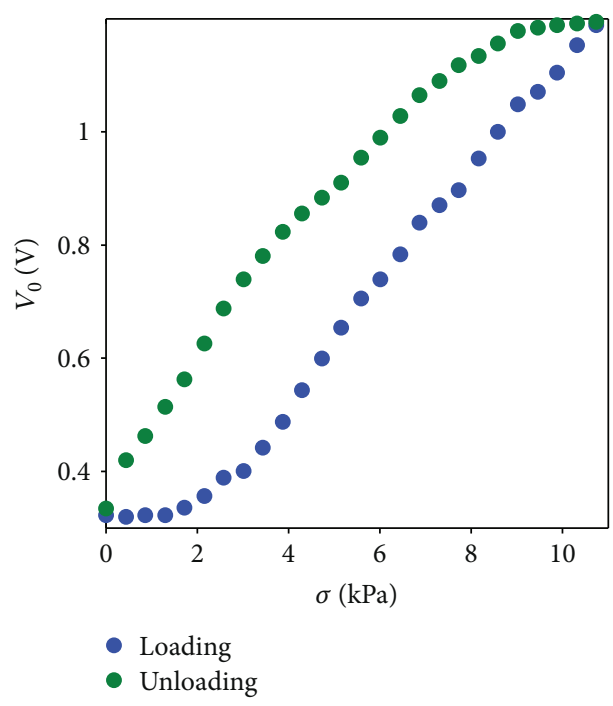

(b)

Figure 19: Experimental data taken from (a) the circuit in Figure 16 and (b) the circuit in Figure 18.

TABLE 4: Repeatability error for the proposed method based on capacitance measurements.

\begin{tabular}{|c|c|c|c|c|c|c|c|c|}
\hline \multirow[t]{2}{*}{ Load } & \multirow{2}{*}{$\begin{array}{c}\text { Sensor } \\
1\end{array}$} & \multicolumn{5}{|c|}{ Data sets } & \multirow{2}{*}{$\begin{array}{c}\mathrm{ER}_{1} \\
2 \%\end{array}$} & \multirow{2}{*}{$\frac{\mathrm{ER}_{2}}{1 \%}$} \\
\hline & & 9 & 8.8 & 8.8 & 9 & 8.8 & & \\
\hline \multirow{3}{*}{$200 \mathrm{~g}$} & 2 & 7.4 & 7.4 & 7.5 & 7.4 & 7.3 & $3 \%$ & $1 \%$ \\
\hline & 3 & 8.5 & 8.6 & 8.6 & 8.6 & 8.5 & $1 \%$ & $1 \%$ \\
\hline & 4 & 6.2 & 6.3 & 6.2 & 6.2 & 6.3 & $2 \%$ & $1 \%$ \\
\hline \multicolumn{7}{|c|}{ Average errors } & $2 \%$ & $1 \%$ \\
\hline \multirow{4}{*}{$400 \mathrm{~g}$} & 1 & 10.5 & 10.5 & 10.6 & 10.7 & 10.7 & $2 \%$ & $1 \%$ \\
\hline & 2 & 10 & 9.9 & 9.8 & 10 & 10 & $2 \%$ & $1 \%$ \\
\hline & 3 & 11.2 & 11.1 & 11.2 & 11.2 & 11.2 & $1 \%$ & $0 \%$ \\
\hline & 4 & 9.6 & 9.9 & 9.7 & 9.7 & 9.6 & $3 \%$ & $1 \%$ \\
\hline \multicolumn{3}{|c|}{ Average errors } & & & & & $2 \%$ & $1 \%$ \\
\hline
\end{tabular}

TABLE 5: Repeatability error for the traditional method based on conductance measurements.

\begin{tabular}{|c|c|c|c|c|c|c|c|c|}
\hline \multirow[t]{2}{*}{ Load } & \multirow{2}{*}{$\begin{array}{c}\text { Sensor } \\
1\end{array}$} & \multicolumn{5}{|c|}{ Data sets } & \multirow{2}{*}{$\frac{\mathrm{ER}_{1}}{22 \%}$} & \multirow{2}{*}{$\frac{\mathrm{ER}_{2}}{9 \%}$} \\
\hline & & 0.277 & 0.248 & 0.225 & 0.225 & 0.233 & & \\
\hline \multirow{3}{*}{$200 \mathrm{~g}$} & 2 & 0.176 & 0.179 & 0.166 & 0.186 & 0.184 & $11 \%$ & $4 \%$ \\
\hline & 3 & 0.245 & 0.252 & 0.246 & 0.258 & 0.25 & $5 \%$ & $2 \%$ \\
\hline & 4 & 0.27 & 0.258 & 0.28 & 0.283 & 0.274 & $9 \%$ & $4 \%$ \\
\hline \multicolumn{7}{|c|}{ Average errors } & $12 \%$ & $5 \%$ \\
\hline \multirow{4}{*}{$400 \mathrm{~g}$} & 1 & 0.418 & 0.437 & 0.365 & 0.335 & 0.38 & $26 \%$ & $11 \%$ \\
\hline & 2 & 0.3 & 0.28 & 0.241 & 0.242 & 0.278 & $22 \%$ & $10 \%$ \\
\hline & 3 & 0.382 & 0.385 & 0.372 & 0.376 & 0.362 & $6 \%$ & $2 \%$ \\
\hline & 4 & 0.4 & 0.422 & 0.4 & 0.404 & 0.386 & $9 \%$ & $3 \%$ \\
\hline \multicolumn{7}{|c|}{ Average errors } & $16 \%$ & $6 \%$ \\
\hline
\end{tabular}

\section{Conclusions}

CPCs exhibit both piezoresistive and piezocapacitive effects due to two factors: quantum tunneling and electrical field confirmation among conductive particles, respectively. Piezoresistive effect has been useful for force sensing due to sensitivity, low invasive profile, and low cost. However, hysteresis phenomenon is observed due to the polymeric behavior of its matrix and this is a concerning drawback worth to be lessen through seeking alternative phenomena in them or modifying the conditioning circuits to gain accuracy although cost increases. Load cells, FSR competitors, are enormously more expensive. Their cost ascends up to USD 500.00 while FSRs' cost roughly reaches not more than USD 25.00. This price gap permits to boost considerably the conditioning circuits' complexity. However, research aims to find out even cheaper alternatives to make them more attractive. Piezocapacitive effect is an alternative phenomenon with the advantage of not having exponential relations that increases hysteresis and therefore, considering piezocapacitance for force sensing is a valid alternative to reduce hysteresis error. Moreover, its nondependence on voltage also lessens repeatability as an additional advantage that contributes to accuracy. Although measuring capacitance is not that straightforward as measuring resistance, it is allowed to boost conditioning circuit complexity due to the aforementioned price gap in comparison to load cells. The proposed method in this paper is the least costly solution in comparison to those found in literature. Current endeavors aim to improve accuracy of force sensing methods to broaden FSR usage at industry. The main goal is to make them measure as good as load cells with considerable cost reduction.

\section{Conflicts of Interest}

The authors declare that they have no conflicts of interest. 


\section{Acknowledgments}

This work was supported by COLCIENCIAS through Francisco José de Caldas Fund (FP44842-335-2015) and by PI/ UAN-2017-613GIBIO and PI/UAN-2017-616GFM grants.

\section{References}

[1] M. Y. Saadeh and M. B. Trabia, "Identification of a forcesensing resistor for tactile applications," Journal of Intelligent Material Systems and Structures, vol. 24, no. 7, pp. 813-827, 2013.

[2] E. R. Komi, J. R. Roberts, and S. J. Rothberg, "Evaluation of thin, flexible sensors for time-resolved grip force measurement," Proceedings of the Institution of Mechanical Engineers, Part C: Journal of Mechanical Engineering Science, vol. 221, no. 12, pp. 1687-1699, 2007.

[3] F. Vecchi, C. Freschi, S. Micera, A. M. Sabatini, P. Dario, and R. Sacchetti, "Experimental evaluation of two commercial force sensors for applications in biomechanics and motor control," in 5th; Annual Conference, International Functional Electrical Stimulation Society, pp. 45-54, Aalborg, Denmark, 2000.

[4] I. Muller, R. de Brito, C. Pereira, and V. Brusamarello, "Load cells in force sensing analysis - theory and a novel application," IEEE Instrumentation \& Measurement Magazine, vol. 13, no. 1, pp. 15-19, 2010.

[5] C. Lebosse, B. Bayle, M. de Mathelin, and P. Renaud, "Nonlinear modeling of low cost force sensors," in 2008 IEEE International Conference on Robotics and Automation, pp. 3437-3442, Pasadena, CA, USA, 2008.

[6] X.-W. Zhang, Y. Pan, Q. Zheng, and X.-S. Yi, “Time dependence of piezoresistance for the conductor-filled polymer composites," Journal of Polymer Science Part B: Polymer Physics, vol. 38, no. 21, pp. 2739-2749, 2000.

[7] A. D. Lantada, P. Lafont, J. L. M. Sanz, J. M. Munoz-Guijosa, and J. E. Otero, "Quantum tunnelling composites: characterisation and modelling to promote their applications as sensors," Sensors and Actuators A: Physical, vol. 164, no. 1-2, pp. 46-57, 2010.

[8] M. Kalantari, J. Dargahi, J. Kovecses, M. G. Mardasi, and S. Nouri, "A new approach for modeling piezoresistive force sensors based on semiconductive polymer composites," IEEE/ASME Transactions on Mechatronics, vol. 17, no. 3, pp. 572-581, 2012.

[9] L. Wang, "Piezoresistive sensor based on conductive polymer composite with transverse electrodes," IEEE Transactions on Electron Devices, vol. 62, no. 4, pp. 1299-1305, 2015.

[10] X. Tan, R. Venkataraman, and P. Krishnaprasad, "Control of hysteresis: theory and experimental results," in SPIE's 8th Annual International Symposium on Smart Structures and Materials, pp. 101-112, Newport Beach, CA, USA, 2001.

[11] J. G. Simmons, "Electric tunnel effect between dissimilar electrodes separated by a thin insulating film," Journal of Applied Physics, vol. 34, no. 9, pp. 2581-2590, 1963.

[12] L. Paredes-Madrid, L. Emmi, E. Garcia, and P. Gonzalez de Santos, "Detailed study of amplitude nonlinearity in piezoresistive force sensors," Sensors, vol. 11, no. 12, pp. 8836-8854, 2011.

[13] S. Stassi, A. Sacco, and G. Canavese, "Impedance spectroscopy analysis of the tunnelling conduction mechanism in piezoresistive composites," Journal of Physics D: Applied Physics, vol. 47, no. 34, article 345306, 2014.

[14] A. Damilano, A. Lince, S. Appendino et al., "Commercial tactile sensors for hand exoskeletons: practical considerations for ultra-low cost and very-low complexity read-out," IEEE Instrumentation Measurement Magazine, vol. 19, no. 5, pp. 49-56, 2016.

[15] L. Wang, "Variations in the capacitance and dielectric constant of multi-wall carbon nanotube filled silicone rubber composite during compressive creep," Composites Science and Technology, vol. 130, pp. 1-8, 2016.

[16] A. Matute, L. Paredes-Madrid, E. Gutierrez, and C. A. P. Vargas, "Characterization of drift and hysteresis errors in force sensing resistors considering their piezocapacitive effect," in 2017 IEEE Sensors, pp. 1-3, Glasgow, UK, 2017.

[17] M. I. Tiwana, S. J. Redmond, and N. H. Lovell, "A review of tactile sensing technologies with applications in biomedical engineering," Sensors and Actuators A: Physical, vol. 179, pp. 17-31, 2012.

[18] S. J. Dempsey, M. Szablewski, and D. Atkinson, "Tactile sensing in human-computer interfaces: the inclusion of pressure sensitivity as a third dimension of user input," Sensors and Actuators A: Physical, vol. 232, pp. 229-250, 2015.

[19] B. Krivopal, "Pressure sensitive ink means, and method of use," US Patent 5,989,700, 1999.

[20] B. Oreper and J. Brenneman, "Pressure sensor," US Patent 6,272,936, 2001.

[21] S. Stassi, V. Cauda, G. Canavese, and C. Pirri, "Flexible tactile sensing based on piezoresistive composites: a review," Sensors, vol. 14, no. 12, pp. 5296-5332, 2014.

[22] S. Stassi, G. Canavese, F. Cosiansi et al., "Smart piezoresistive tunnelling composite for flexible robotic sensing skin," Smart Materials and Structures, vol. 22, no. 12, article 125039, 2013.

[23] D. Bloor, K. Donnelly, P. J. Hands, P. Laughlin, and D. Lussey, "A metal-polymer composite with unusual properties," Journal of Physics D: Applied Physics, vol. 38, no. 16, pp. 28512860, 2005.

[24] J. Dabling, A. Filatov, and J. Wheeler, "Static and cyclic performance evaluation of sensors for human interface pressure measurement," in 2012 Annual International Conference of the IEEE Engineering in Medicine and Biology Society, pp. 162-165, San Diego, CA, USA, 2012.

[25] W. Luheng, D. Tianhuai, and W. Peng, "Influence of carbon black concentration on piezoresistivity for carbon-black-filled silicone rubber composite," Carbon, vol. 47, no. 14, pp. 31513157, 2009.

[26] F. Mainardi and G. Spada, "Creep, relaxation and viscosity properties for basic fractional models in rheology," The European Physical Journal Special Topics, vol. 193, no. 1, pp. 133-160, 2011.

[27] D. de Tommasi, G. Puglisi, and G. Zurlob, "Hysteresis in electroactive polymers," European Journal of Mechanics A/Solids, vol. 48, pp. 16-22, 2014.

[28] F. Vogel, S. Göktepe, P. Steinmann, and E. Kuhl, "Modeling and simulation of viscous electro-active polymers," European Journal of Mechanics - A/Solids, vol. 48, pp. 112-128, 2014.

[29] J.-S. Kim and G.-W. Kim, "Hysteresis compensation of piezoresistive carbon nanotube/polydimethylsiloxane compositebased force sensors," Sensors, vol. 17, no. 12, p. 229, 2017. 
[30] J. C. Fisher and I. Giaever, "Tunneling through thin insulating layers," Journal of Applied Physics, vol. 32, no. 2, pp. 172-177, 1961.

[31] L. Paredes-Madrid, C. A. Palacio, A. Matute, and C. A. Parra Vargas, "Underlying physics of conductive polymer composites and force sensing resistors (fsrs) under static loading conditions," Sensors, vol. 17, no. 12, 2017.

[32] G. R. Ruschau, S. Yoshikawa, and R. E. Newnham, "Resistivities of conductive composites," Journal of Applied Physics, vol. 72, no. 3, pp. 953-959, 1992.

[33] A. Mikrajuddin, F. G. Shi, H. Kim, and K. Okuyama, "Sizedependent electrical constriction resistance for contacts of arbitrary size: from Sharvin to Holm limits," Materials Science in Semiconductor Processing, vol. 2, no. 4, pp. 321-327, 1999.

[34] A. M. Almassri, W. Z. Wan Hasan, S. A. Ahmad et al., "Pressure sensor: state of the art, design, and application for robotic hand," Journal of Sensors, vol. 2015, Article ID 846487, 12 pages, 2015.

[35] L. Wang and Y. Li, "A review for conductive polymer piezoresistive composites and a development of a compliant pressure transducer," IEEE Transactions on Instrumentation and Measurement, vol. 62, no. 2, pp. 495-502, 2013.

[36] L. Paredes-Madrid, A. Matute, and A. Peña, "Framework for a calibration-less operation of force sensing resistors at different temperatures," IEEE Sensors Journal, vol. 17, no. 13, pp. 41334142, 2017.

[37] F. S. Corporation, LM 555 Single Timer, On Semiconductor, Aurora, CO, USA, 2013.

[38] L. Paredes-Madrid, A. Matute, J. O. Bareño, C. A. Parra Vargas, and E. I. Gutierrez Velásquez, "Underlying physics of conductive polymer composites and force sensing resistors (FSRs). A study on creep response and dynamic loading," Materials, vol. 10, no. 12, 2017.

[39] M. Faraz, Z. Salcic, and K. Wang, "Analysis and selection of the force sensitive resistors for gait characterization," in 2015 6th International Conference on Automation, Robotics and Applications (ICARA), pp. 370-375, Queenstown, New Zealand, 2015. 


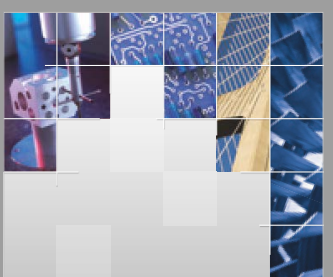

\section{Enfincering}
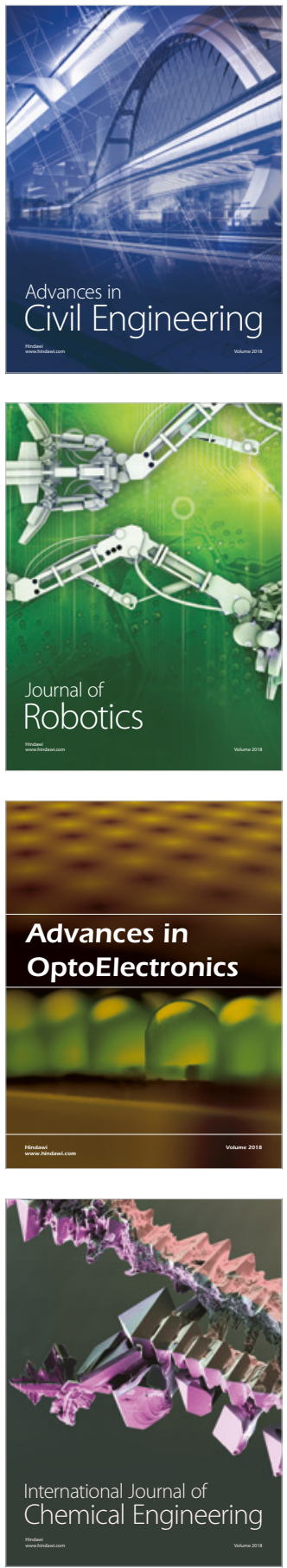

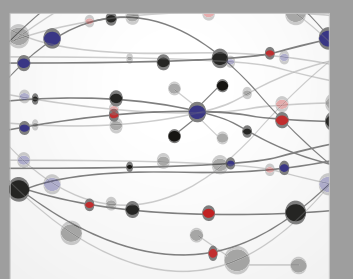

\section{Rotating \\ Machinery}

The Scientific World Journal

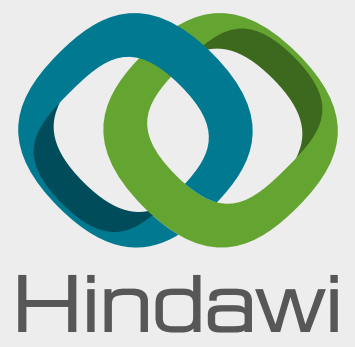

Submit your manuscripts at

www.hindawi.com
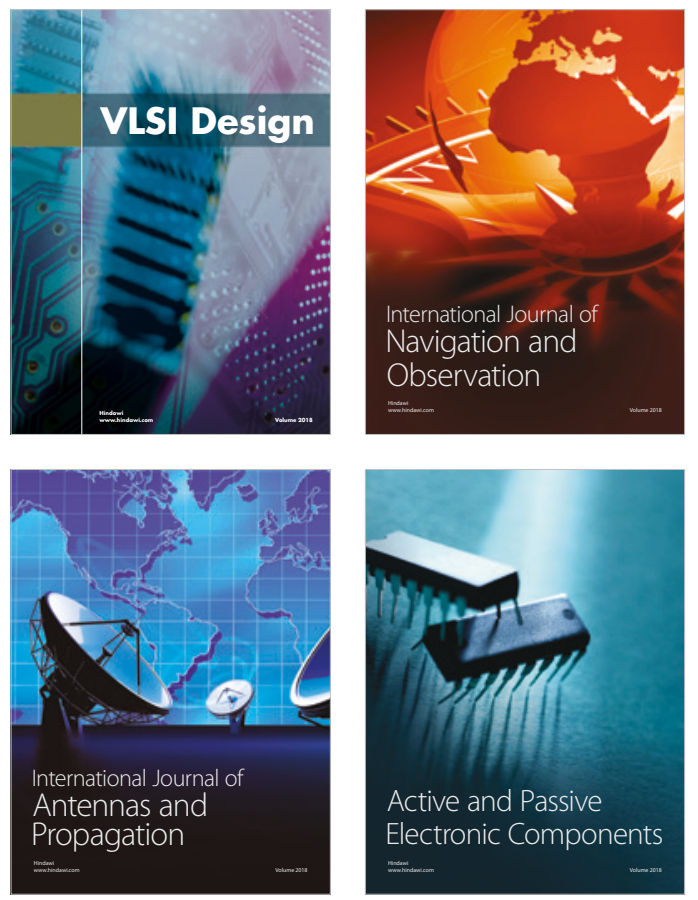
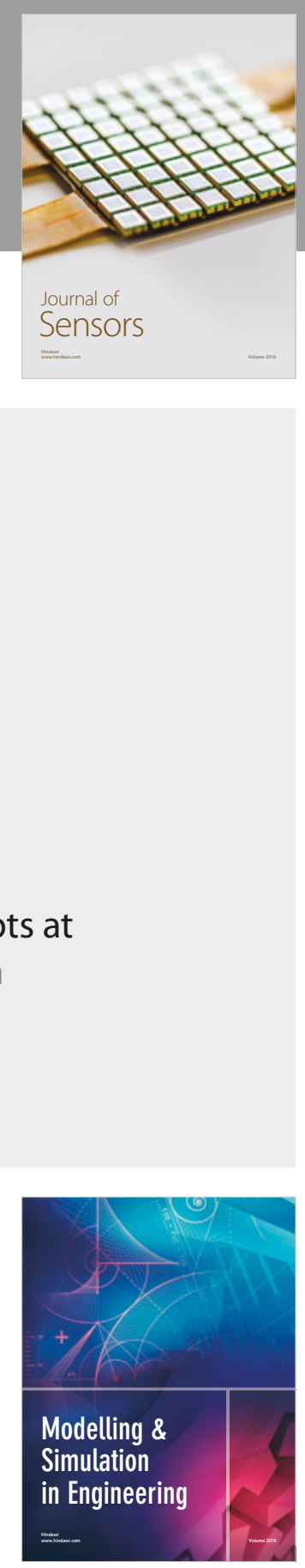

\section{Advances \\ Multimedia}
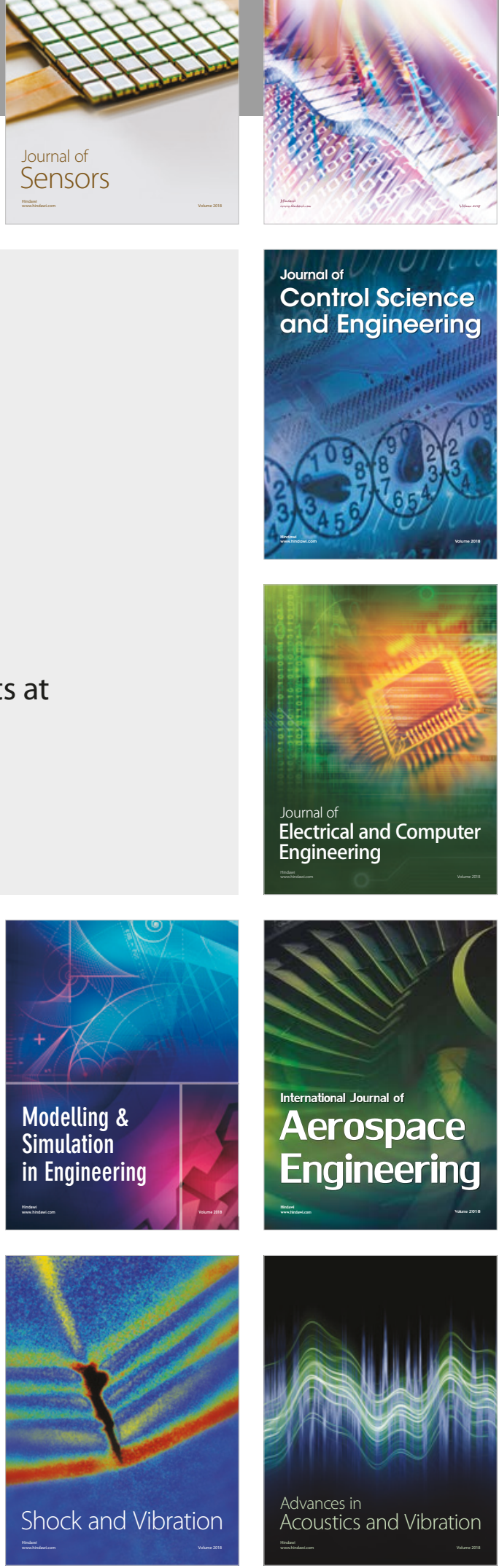